\title{
A non-contact acoustic pressure-based method for load identification in acoustic-structural interaction system with non-probabilistic uncertainty
}

\author{
Z. C. He ${ }^{1,3}, X$. Y. Lin ${ }^{1}$, Eric $\mathrm{Li}^{2 *}$ \\ ${ }^{1}$ State Key Laboratory of Advanced Design and Manufacturing for Vehicle Body, Hunan \\ University, Changsha, 410082 P. R. China \\ ${ }^{2}$ School of Science, Engineering \& Design, Teesside University, Middlesbrough, UK \\ ${ }^{3}$ Guangxi Key Laboratory of Automobile Components and Vehicle Technology, Guangxi University \\ of Science and Technology
}

\begin{abstract}
A non-contact acoustic pressure-based method is proposed for load identification in the acoustic structure interaction system involving non-probabilistic uncertainty. The forward problem for load identification is established through the discretized convolution integral relationship of the dynamic loads and the Green's kernel function matrix of the system. The inverse process is constructed by using truncated single value decomposition approach in order to overcome the ill-posedness of the global kernel function matrix. In this work, two non-probabilistic models including ellipsoid model and interval model are proposed to quantify the effects of the system uncertainty. Several numerical examples are investigated to verify the effectiveness of the present methods. The results show that the non-contact acoustic pressure-based method with great convenience for dynamic load identification is accurate and effective. For the system with non-probabilistic uncertainty, the ellipsoid model and interval model are the proper choices to identify the bounds with knowing only the extreme values of the parameters. Moreover, the load bounds derived from the ellipsoid model are more reliable than those derived from the interval model.
\end{abstract}

Key Words: Load identification; Acoustic-structure interaction; Non-contact; Non-probabilistic uncertainty;

\footnotetext{
2* School of Science, Engineering \& Design, Teesside University, Middlesbrough, UK, Email address: ericsg2012@gmail.com (Eric Li)
} 


\section{Introduction}

In structural dynamic problems, load identification has become a key approach to satisfy the engineering design, such as vibration and noise control, reliability analysis, and structural optimization [1]. Unfortunately, due to technical or economic limitations, dynamic load cannot be directly and successfully measured in many practical applications, such as engine and road excitations applied to vehicle, wind load applied to tall building, interaction load between railway track and train wheel [2]. For this reason, it is important and necessary to improve the load identification techniques.

The dynamic load identification can be classified as frequency-domain methods [3-6] and time-domain methods [7-10]. In the frequency domain, the exciting load spectrums are determined by the relationship between the frequency response functions and the measured responses spectrums during operation in modal coordinate system, and the load history is then derived through the modal coordinate transformation. In the time domain, with the help of the kinematics equations, the methods formulated mainly depend on the convolution relationship between the load and the system response so as to obtain the time history of load. Compared to the frequency-domain methods, time domain methods are able to identify the load directly without transforming errors and thus are suitable for any types of load.

In both frequency-domain and time-domain, the methods for load identification have undergone a rapid development. Giansante [11] used the acceleration responses to identify 
the load in the helicopter model. Hillary [12] applied the measurement of the strain gauges to determine the load and improved the accuracy of the identification in the low frequency region. Yen and $\mathrm{Wu}[13]$ employed the strain responses at certain points on a rectangle isotropic plate to identify both the location and time history of a transverse impact load. Using the acceleration and velocity response, Thite [3, 4] illustrated different kinds of regularization methods to improve the inverse determination of force. Based on structural displacement, Liu and Han [7] presented inverse procedures to identify both concentrated and extended line load in time domain. In summary, the methods of load identification in these works are mainly based on the vibration-based response namely displacements, velocities, strains or accelerations. Generally, all of these responses need to be measured in a contact way and the measurements require attaching transducers to structure surfaces in order to sense and receive the structural signal. However, the contact measurement may lead to the destruction of the vibration structure. On the other hand, the weight of the transducers used in the contact measurement can change the vibration of the structure especially the vibration of the thin-walled structure. In many applications, such as the acoustic structure interaction (ASI) system $[14,15]$ and thermal acoustic interaction (TAI) system, non-contact measurement is more appropriate than the contact approach as the operating condition with potential damage in contact transducers or the attachments is prohibitive [16].

Recently, the non-contact acoustic technique has attracted lots of interest among many researchers. One of the most important applications of acoustics is to determine the 
load location, load distribution, the vibration reconstruction of the interface between fluid and solid, and the history of sound waves produced by a vibrating source. In these usages, the acoustic holography of close points is a very helpful tool to identify a source of sound and measure vibration of boundary surface shared between fluid and solid [17-19]. In addition to acoustic holography [17, 19], other methods such as acoustic array [20], acoustic transfer matrix method [21] and acoustic inverse [22] have been employed to realize the various tasks of the inverse problems. In sum, all of these methods used the non-contact acoustic pressure to solve the inverse problems. However, there is limitation in identifying the loads acting on the structure as either the process is too complicated or the loads cannot be identified stably and efficiently. Thus, it is still necessary to develop an efficient strategy to identify the loads by using the non-contact acoustic pressure.

In addition, in the design system with uncertainty issues, the above-mentioned methods for the load identification of the ASI system are only limited in the deterministic parameters. However, the uncertainties widely exist in material properties, external load, boundary conditions, measurement and manufacture [23]. Quantifying these effects from the uncertainties in load identification is indispensable in practical engineering applications. Probability model [24-26] is still considered as the most widely used mathematical model to deal with the uncertainties in engineering application. In the probabilistic model, the uncertain parameter is treated as random variable whose probability density function (PDF) is well defined in its variational range. Fuzzy-set model $[27,28]$ is another category to describe the parametric uncertainties with limited objective 
information in a non-probabilistic way. In fuzzy-set model, the uncertain parameters are described as the variables with known fuzzy membership functions (FMFs). The above-two models own a common characteristic that the calculated model needs the precise information of the uncertain parameter.

However, obtaining the precise information is challenging in the practical engineering because sufficient statistical data are required to construct the PDF or the FMFs of uncertain parameters. To overcome this drawback, the non-probability convex method [29] has emerged as an alternative approach to deal with the uncertain parameters with limited information not necessarily knowing the PDF or FMFs. Presently, two kinds of non-probabilistic convex model namely ellipsoid model [30, 31] and interval model [32-34] are widely used. In the interval model, the uncertain parameters are assumed to be independent with each other and they form a multidimensional cuboid. In this case, these interval parameters are called "independent interval variables" and each of them belongs to an interval in the non-probabilistic convex model. In the ellipsoid model, the uncertain variables are assumed to be mutually dependent and the uncertainty domain is described by a multidimensional ellipsoid. Liu [35] firstly used the interval model to identify the load acting on the uncertain structural plate, where only the lower and upper bounds of the uncertain parameters are required. However, there is a certain degree of limitation by using the interval method to identify the load. As the load bounds calculated by the interval model are too narrow, it may produce unreliable results and cannot be directly used in engineering practice. 
In order to address the above problems, a novel, non-contact acoustic pressure-based method using Green's kernel function method combined with non-probabilistic theory is proposed to identify the load acting on the structure for the ASI system. The main advantages of the proposed method are that: (i) the acoustic pressure that is measured through the non-contact way can replace the structural responses and therefore increases the convenience of the inverse problems; and (ii) the non-probabilistic uncertainties of the ASI system are further taken into consideration for load identification in order to satisfy the practical condition.

The remainder of the paper is organized as follows: the forward problem of load identification based on sound pressure through acoustic structure interaction is constructed in Section 2. Simultaneously, truncated single value decomposition as a proper regularization approach is employed to ensure the stable solutions such that the ill-posedness arisen in load identification can be efficiently overcome. Section 3 applies the non-probabilistic ellipsoid model for the uncertain parameters where the effect of the structural uncertainty on the load identification is quantitatively described. To compare with the ellipsoid model, the identification deduction by interval model is also given in Section 3. Several numerical applications are analyzed in Section 4 and conclusions are finally summarized in Section 5.

\section{Construction of the forward problem based on acoustic pressure}

\subsection{Mathematical model of ASI}


Given the interaction between the vibrating structure and the acoustic field, the coupled structural-acoustic system has been widely used in submarine, automobile body and aircraft. The structural dynamic equation of motion is expressed as [36-40]:

$$
\mathbf{M}_{\mathrm{s}} \ddot{\mathbf{u}}+\mathbf{C}_{\mathrm{s}} \dot{\mathbf{u}}+\mathbf{K}_{\mathrm{s}} \mathbf{u}=\mathbf{F}_{\mathrm{s}}
$$

where $\mathbf{u}=\left\{u_{1}, u_{2}, \ldots, u_{i}, \ldots, u_{M}\right\}^{\mathrm{T}}$ stands for the nodal displacement vector of the structural responses; $\mathbf{M}_{\mathrm{s}}$ is the structural mass matrix, $\mathbf{C}_{\mathrm{s}}$ is the structural damping matrix, $\mathbf{K}_{\mathrm{s}}$ is the structural stiffness matrix, and $\mathbf{F}_{\mathrm{s}}$ is the vector of structure nodal load, and given in Eq. (2):

$$
\begin{gathered}
\mathbf{M}_{\mathrm{s}}=\sum_{i=1}^{\text {Mcell }} \int_{\Omega_{\mathrm{s}}} \rho_{\mathrm{s}} \mathbf{N}_{\mathrm{s}}^{\mathrm{T}} \mathbf{N}_{\mathrm{s}} \mathrm{d} \Omega, \mathbf{C}_{\mathrm{s}}=\sum_{i=1}^{\text {Mcell }} \int_{\Gamma_{\mathrm{s}}} \mathbf{N}_{\mathrm{s}}^{\mathrm{T}} \mathbf{N}_{\mathrm{s}} \varsigma \mathrm{d} \Gamma, \\
\mathbf{K}_{\mathrm{s}}=\sum_{i=1}^{\text {Mcell }} \int_{\Omega_{\mathrm{s}}} \mathbf{B}_{\mathrm{s}}^{\mathrm{T}} \mathbf{D}_{\mathrm{s}} \mathbf{B}_{\mathrm{s}} \mathrm{d} \Omega, \mathbf{F}_{\mathrm{s}}=\sum_{i=1}^{\text {Mcell }} \int_{\Omega_{\mathrm{s}}} \mathbf{N}_{\mathrm{s}}^{\mathrm{T}} \tau \mathrm{d} \Omega,
\end{gathered}
$$

where the summation represents the assembly process of the structural system matrices and vectors using the finite element method; Mcell is the total number of the structural elements; $\rho_{\mathrm{s}}, \mathbf{N}_{\mathrm{s}}, \varsigma, \mathbf{B}_{\mathrm{s}}, \mathbf{D}_{\mathrm{s}}$, and $\mathbf{F}_{\mathrm{s}}$ denote the structural density, the structural shape function, the damping factor, the structural strain matrix, the structural elastic matrix and the force vector, respectively. $\tau$ is the surface traction force arising from the acoustic part. The superscript $\mathrm{T}$ denotes the transpose of the relevant matrix. $\Omega_{\mathrm{s}}$ and $\Gamma_{\mathrm{s}}$ are the structure domain and structure boundary respectively.

The discrete system equation of the acoustic domain can be written in the following matrix form [41]:

$$
\mathbf{M}_{\mathrm{a}} \ddot{\mathbf{p}}+\mathbf{C}_{\mathrm{a}} \dot{\mathbf{p}}+\mathbf{K}_{\mathrm{a}} \mathbf{p}-\mathbf{F}_{\mathrm{sa}}=0
$$


where:

$$
\begin{array}{ll}
\mathbf{K}_{\mathrm{a}}=\sum_{i=1}^{\text {Ncell }} \int_{\Omega}\left(\nabla \mathbf{N}_{\mathrm{a}}\right)^{\mathrm{T}} \nabla \mathbf{N}_{\mathrm{a}} \mathrm{d} \Omega=\sum_{i=1}^{\text {Ncell }} \int_{\Omega} \mathbf{B}_{\mathrm{a}}^{\mathrm{T}} \mathbf{B}_{\mathrm{a}} \mathrm{d} \Omega & \text { The acoustic stiffness matrix } \\
\mathbf{M}_{\mathrm{a}}=\sum_{i=1}^{\text {Ncell }} \int_{\Omega} \mathbf{N}_{\mathrm{a}}^{\mathrm{T}} \mathbf{N}_{\mathrm{a}} \mathrm{d} \Omega & \text { The acoustic mass matrix } \\
\mathbf{C}_{\mathrm{a}}=\sum_{i=1}^{N \text { cell }} \int_{\Gamma_{A}} \rho_{\mathrm{a}} \mathbf{N}_{\mathrm{a}}^{\mathrm{T}} \mathbf{N}_{\mathrm{a}} A_{n} \mathrm{~d} \Gamma & \text { The acoustic damping matrix } \\
\mathbf{F}_{\mathrm{sa}}=\sum_{i=1}^{N \text { Neell }} \int_{\Omega_{\mathrm{sa}}} \mathbf{N}_{\mathrm{a}}^{\mathrm{T}} \rho_{\mathrm{a}} \ddot{\mathrm{a}} \mathrm{a} \mathrm{d} \Gamma & \text { The vector of nodal acoustic forces } \\
\mathbf{p}=\left\{p_{1}, p_{2}, \ldots, p_{j}, \ldots, p_{n}\right\}^{\mathrm{T}} & \text { Nodal acoustic pressure in the domain }
\end{array}
$$

where the summation represents the assembly process of the acoustic system matrices and vectors using the finite element method; Ncell is the total number of the acoustic elements; $\rho_{\mathrm{a}}$ denotes the density of the fluid in the acoustic field; $\mathbf{N}_{\mathrm{a}}$ and $\mathbf{B}_{\mathrm{a}}$ are the shape function and the strain matrix of the fluid; $A_{n}$ is the admittance coefficient that models the structural damping on the normal impedance boundary; $u_{\mathrm{a}}$ is the displacement of the fluid part.

In the coupled ASI system, this work only considers the displacement continuity condition in the interface. Therefore, the vector of nodal acoustic forces can be rewritten as:

$$
\begin{aligned}
& \text { Displacement continuity: } u_{i} \cdot \mathbf{n}_{\mathrm{a}}=u_{\mathrm{a}} \cdot \mathbf{n}_{\mathrm{a}} \\
& \mathbf{F}_{\mathrm{sa}}=\int_{\Omega_{\mathrm{sa}}} \mathbf{N}_{\mathrm{a}}^{\mathrm{T}} \rho_{\mathrm{a}} \ddot{u_{\mathrm{a}}} \mathrm{d} \Gamma=\rho_{\mathrm{a}}\left(\int_{\Omega_{\mathrm{sa}}} \mathbf{N}_{\mathrm{a}}^{\mathrm{T}} \mathbf{n}_{\mathrm{a}} \mathbf{N}_{\mathrm{s}} \mathrm{d} \Gamma\right) \ddot{\mathbf{u}}
\end{aligned}
$$

where $\mathbf{n}_{\mathrm{a}}$ denotes the normal vector external to the coupled interface, and $\Omega_{\mathrm{sa}}$ is the coupled interface; $\sigma$ is the stress of the solid part. Under the external force, the coupled matrix equation for the ASI problem without considering the acoustic source excitation 
can be deduced by substituting Eqs. (4) into (3) and combining Eqs. (1) and (3):

$$
\left[\begin{array}{cc}
\mathbf{M}_{\mathrm{s}} & 0 \\
-\rho_{\mathrm{a}} \mathbf{H}^{\mathrm{T}} & \mathbf{M}_{\mathrm{a}}
\end{array}\right]\left\{\begin{array}{c}
\ddot{\mathbf{u}} \\
\ddot{\mathbf{p}}
\end{array}\right\}+\left[\begin{array}{cc}
\mathbf{C}_{\mathrm{s}} & 0 \\
0 & \mathbf{C}_{\mathrm{a}}
\end{array}\right]\left\{\begin{array}{c}
\dot{\mathbf{u}} \\
\dot{\mathbf{p}}
\end{array}\right\}+\left[\begin{array}{cc}
\mathbf{K}_{\mathrm{s}} & 0 \\
0 & \mathbf{K}_{\mathrm{a}}
\end{array}\right]\left\{\begin{array}{l}
\mathbf{u} \\
\mathbf{p}
\end{array}\right\}=\left\{\begin{array}{c}
\mathbf{F}_{\mathrm{s}} \\
0
\end{array}\right\}
$$

where $\mathbf{M}_{\mathrm{s}}, \mathbf{C}_{\mathrm{s}}$ and $\mathbf{K}_{\mathrm{s}}$ are the mass, damping and stiffness matrices from structural domain; $\mathbf{M}_{\mathrm{a}}, \mathbf{C}_{\mathrm{a}}$ and $\mathbf{K}_{\mathrm{a}}$ are the mass, damping and stiffness matrices from acoustic domain; $\mathbf{F}_{\mathrm{s}}$ is the vector of structure nodal load; $\rho_{\mathrm{a}}$ is the acoustic medium density; $\mathbf{u}$ and $\mathbf{p}$ are the structural displacement vector and the acoustic pressure vector, respectively. $\mathbf{H}$ represents the coupled matrix on the interface, and it is given by:

$$
\mathbf{H}=\int_{\Omega_{\mathrm{sa}}} \mathbf{N}_{\mathrm{a}}^{\mathrm{T}} \mathbf{n}_{\mathrm{a}} \mathbf{N}_{\mathrm{s}} \ddot{\mathbf{u}} \mathrm{d} \Gamma
$$

Obviously, it can be seen from Eq. (5) that while the solving process of the structure response is decoupled in the structural domain, the solving in the acoustic domain is coupled. Since the acoustic equation is affected by the structure responses on the interface, Eq. (5) can be divided into two individual portions; therefore, the interaction response analysis can transfer into two steps: the first step is to compute the structural response, and the second step is to solve the pressure response.

\subsection{Forward problem of load identification based on sound pressure}

Traditionally, the methods of load identification use the measurement of the structural response, which can be acceleration, velocity, displacement, stress, and strain, to realize the inverse process. Initially, the single point load is identified through the pressure-based method. Given a linear and time-invariant coupled ASI system, the history of the structural response at an arbitrary time point can be derived through the convolution integral of the 
load function and the corresponding Green's kernel in time domain [10, 42].

$$
u_{i}(x, t)=\int_{0}^{t} F(\tau) g(x, t-\tau) \mathrm{d} \tau
$$

where $u_{i}(x, t)$ is the traditional structural measured response of the given response point $x$ in the structure domain, $g_{i}(x, t)$ is the Green's function, representing the dynamic behaviors within the excitation of the unit-pulse force; and $F_{i}(t)$ is the unknown point load acting on a certain location of the structure.

As Eq. (3) can be divided into two separate parts, the following form can be yielded:

$$
\mathbf{M}_{\mathrm{a}} \ddot{\mathbf{p}}+\mathbf{C}_{\mathrm{a}} \dot{\mathbf{p}}+\mathbf{K}_{\mathrm{a}} \mathbf{p}=\rho_{\mathrm{a}} \mathbf{H}^{\mathrm{T}} \ddot{\mathbf{u}}
$$

where $\mathbf{u}=\left\{u_{1}, u_{2}, \ldots, u_{i}, \ldots, u_{M}\right\}^{\mathrm{T}}$, and $u_{i}$ defined in Eq. (7) is the structural response of the $i$-th node on the interface.

It is noted that the acoustic pressure can be produced directly with the force action. Therefore, the convolution integral relationship between the force and the acoustic pressure can be further expressed as the form:

$$
p_{j}(y, t)=\int_{0}^{t} F(\tau) G(y, t-\tau) \mathrm{d} \tau
$$

where $G$ denotes the Green's function from the excitation point of the structure to the measured point $y$ of the acoustic cavity under the unit-pulse force; $p_{j}(y, t)$ is the acoustic pressure; $F(t)$ is the acting load. It should be emphasized that the generation of the acoustic pressure response is due to the structure acoustic interaction from Eq. (9); the measurement for the acoustic pressure is more convenient since non-contact approach is applied. In addition, Eq. (9) is an alternative approach for the computation of the ASI 
system without calculating the coupled Eq. (5). By discretizing Eq. (9) into $m$ equally spaced sample points in the time domain, the continuous convolution integral Eq. (9) is transformed into a discrete matrix form according to the characteristic of the pulse function [7]:

$$
\left\{\begin{array}{c}
p\left(t_{1}\right) \\
p\left(t_{2}\right) \\
\vdots \\
p\left(t_{i}\right) \\
\vdots \\
p\left(t_{m}\right)
\end{array}\right\}=\left[\begin{array}{cccccc}
G\left(t_{1}\right) & 0 & 0 & 0 & 0 & 0 \\
G\left(t_{2}\right) & G\left(t_{1}\right) & 0 & 0 & 0 & 0 \\
\cdots & G\left(t_{2}\right) & \ddots & 0 & 0 & 0 \\
G\left(t_{i}\right) & \cdots & \ddots & G\left(t_{1}\right) & 0 & 0 \\
\vdots & \cdots & \cdots & G\left(t_{2}\right) & \ddots & 0 \\
G\left(t_{m}\right) & G\left(t_{m-1}\right) & \cdots & G\left(t_{3}\right) & G\left(t_{2}\right) & G\left(t_{1}\right)
\end{array}\right]\left\{\begin{array}{c}
F_{0} \\
F_{1} \\
\vdots \\
F_{i-1} \\
\vdots \\
F_{m-1}
\end{array}\right\}
$$

where $p\left(t_{i}\right)$ and $G\left(t_{i}\right)$ represent the measured acoustic response and the Green kernel function response, respectively; $F_{i-1}$ is the exciting load at time point $t_{i}=(i-1) \Delta t$ $(i=1,2, \ldots, m)$; It should be noted that since the ASI system is stationary before load excitation, the values of acoustic pressure response and the Green's kernel function at the initial time are both equal to zero. Then, Eq. (10) can be expressed as the matrix form as:

$$
\mathbf{P}=\mathbf{G F}
$$

where

$$
\begin{aligned}
& \mathbf{P}=\left\{p\left(t_{1}\right), p\left(t_{2}\right), \ldots, p\left(t_{i}\right), \ldots, p\left(t_{m}\right)\right\}^{\mathrm{T}} \\
& \mathbf{F}=\left\{F\left(t_{1}\right), F\left(t_{2}\right), \ldots, F\left(t_{i}\right), \ldots, F\left(t_{m}\right)\right\}^{\mathrm{T}} \\
& \mathbf{G}=\left[\begin{array}{cccccc}
G\left(t_{1}\right) & 0 & 0 & 0 & 0 & 0 \\
G\left(t_{2}\right) & G\left(t_{1}\right) & 0 & 0 & 0 & 0 \\
\ldots & G\left(t_{2}\right) & \ddots & 0 & 0 & 0 \\
G\left(t_{i}\right) & \ldots & \ddots & G\left(t_{1}\right) & 0 & 0 \\
\vdots & \ldots & \cdots & G\left(t_{2}\right) & \ddots & 0 \\
G\left(t_{m}\right) & G\left(t_{m-1}\right) & \cdots & G\left(t_{3}\right) & G\left(t_{2}\right) & G\left(t_{1}\right)
\end{array}\right]
\end{aligned}
$$

where $\mathbf{P}$ and $\mathbf{F}$ are the acoustic pressure vector and the load vector to be identified at 
time point $t_{i}$. $\mathbf{G}$ is the Green's kernel function matrix at time point $t_{i}$. Theoretically, it can be seen from Eq. (10) that the acoustic pressure can be derived directly with the excitation force in a different way from Eq. (5). Moreover, Eq. (11) provides an alternative response i.e. sound pressure for the load identification. As the replaced acoustic pressure can be measured in a non-contact way, it is more convenient for the load identification.

Eq. (10) can realize the identification of a single point load, however, there always exists the application that the multiple points load need to be identified. As the ASI system is treated as a linear and time-invariant system, the total acoustic pressure of the ASI system can be the linear superposition of each single load. Based on Eq. (10), the multiple points load problem can be written as:

$$
\left\{\begin{array}{c}
\mathbf{P}_{1} \\
\mathbf{P}_{2} \\
\vdots \\
\mathbf{P}_{\mathrm{N}}
\end{array}\right\}=\left[\begin{array}{cccc}
\mathbf{G}_{11} & \mathbf{G}_{12} & \cdots & \mathbf{G}_{1 \mathrm{M}} \\
\mathbf{G}_{21} & \mathbf{G}_{22} & \cdots & \mathbf{G}_{2 \mathrm{M}} \\
\vdots & \vdots & \ddots & \vdots \\
\mathbf{G}_{\mathrm{N} 1} & \mathbf{G}_{\mathrm{N} 2} & \cdots & \mathbf{G}_{\mathrm{NM}}
\end{array}\right]\left\{\begin{array}{c}
\mathbf{F}_{1} \\
\mathbf{F}_{2} \\
\vdots \\
\mathbf{F}_{\mathrm{M}}
\end{array}\right\}
$$

where $\mathrm{N}$ stands for the number of the acoustic pressure response points, $\mathrm{M}$ is the number of the multiple points loads; each component of $\mathbf{P}_{j}$ and $\mathbf{F}_{i}$ in Eq. (12) denotes a vector which is defined in Eq. (11); $\mathbf{G}_{j i}$ is the Green's kernel function matrix which represents the acoustic pressure response function from the location of the acting point load $\mathbf{F}_{i}$ to the location of the response point $\mathbf{P}_{j}$. It should be emphasized that in order to reduce the load identification error in the multiple points load identification problem, the number of the acoustic pressure response is generally larger than the number of the point loads [4]. Based on Eqs. (10) and (12), the forward problem of load identification including the 
single point load and the multiple points load can be formed into Eq. (11).

Essentially, Eq. (11) is the forward model for load identification based on acoustic pressure using Green's kernel function method (GKFM). It is well known that the Green's kernel function matrix in Eq. (11) has two obvious characteristics [10]. One is that all the components at the upper triangular part are zero. The second is that the Green's function matrix $\mathbf{G}$ is generally ill-conditioned. Those two characteristics lead to high ill-posedness of the global kernel function matrix and are herein not suitable for load identification through direct matrix inverse operation. Therefore, the regularization approach is used to deal with the aforementioned two drawbacks.

\subsection{Regularization approach}

In practical engineering, the acoustic pressure response in Eq. (11) is obtained through the non-contact measurement. Obviously, the measurement noise cannot be avoided. Noisy sound pressure undoubtedly leads to the ill-posedness of the identified process. To overcome this drawback, the regulation methods namely truncated single value decomposition (TSVD) is herein briefed. Considering the unavoidable noisy data in the measurement of $\mathbf{P}$, the practical acoustic responses in Eq. (11) can be further expressed as:

$$
\mathbf{P}=\mathbf{G F}+\mathbf{e r r}
$$

where err is the noisy data in the form of column vector; $\mathbf{P}$ is the acoustic pressure containing noise. Using the single value decomposition (SVD) for Green's function matrix, the decomposed matrix can be described in the form of: 


$$
\begin{gathered}
\mathbf{G}=\mathbf{W} \sum \mathbf{V}^{\mathrm{T}}=\mathbf{W} \operatorname{Diag}\left(\sigma_{i}\right) \mathbf{V}^{\mathrm{T}}=\sum_{i=1}^{m} \mathbf{w}_{i} \sigma_{i} \mathbf{V}_{i}^{\mathrm{T}} \\
\sum=\left[\begin{array}{c}
\operatorname{diag}\left(\sigma_{1}, \sigma_{2}, \ldots, \sigma_{m}\right) \\
{[0]}
\end{array}\right], \quad \sigma_{1} \geq \sigma_{2} \geq \cdots \geq \sigma_{m} \geq 0
\end{gathered}
$$

where $\mathbf{W}=\left(\mathbf{w}_{1}, \mathbf{w}_{2}, \ldots, \mathbf{w}_{m}\right), \quad \mathbf{V}=\left(\mathbf{v}_{1}, \mathbf{v}_{2}, \ldots, \mathbf{v}_{m}\right)$ represent the left and right singular matrix of $\mathbf{G}$, respectively, $\mathbf{w}_{i}, \mathbf{v}_{i}$ are the $i$-th column vector of $\mathbf{W}$ and $\mathbf{V}$ respectively, $\sigma_{1}, \sigma_{2}, \ldots, \sigma_{m}$ are non-negative singular values arranged in descending order. Besides, these two orthogonal vectors $\mathbf{W}$ and $\mathbf{V}$ have the following relationship:

$$
\begin{gathered}
\mathbf{w}_{i}^{\mathrm{T}} \mathbf{w}_{j}=\delta_{i j}, \quad \mathbf{v}_{i}^{\mathrm{T}} \mathbf{v}_{j}=\delta_{i j} \\
\mathbf{G}_{i}=\sigma_{i} \mathbf{w}_{i}, \mathbf{G}^{\mathrm{T}} \mathbf{w}_{i}=\sigma_{i} \mathbf{v}_{i}
\end{gathered}
$$

Then the load to be identified $\mathbf{F}_{n o i}$ is determined by using the inverse relationship between noisy responses $\mathbf{P}_{n o i}$ and Green's function $\mathbf{G}$ as follows:

$$
\mathbf{F}_{n o i}=\mathbf{G}^{-1} \mathbf{P}_{n o i}=\mathbf{V} \operatorname{Diag}\left(\sigma_{i}^{-1}\right) \mathbf{W}^{\mathrm{T}} \cdot \mathbf{P}^{n o i}=\mathbf{F}+\sum_{i=1}^{m} \sigma_{i}^{-1}\left(\mathbf{w}_{i}^{\mathrm{T}} \cdot \eta_{i}\right) \mathbf{v}_{i}
$$

where $\eta_{i}$ is the $i$-th element of err, $\mathbf{F}$ refers to exact dynamic load (i.e. the actual excitation load acting on the structural part of the coupled model through the finite element method).

It is obviously seen that the success of this formulation largely depends on the accuracy of the matrix inversion. In other word, the identification error is proportional to the reciprocal of $\sigma_{i}$. If matrix $\mathbf{G}$ is ill-conditioned, the ratio between the largest and smallest singular value tends to be high. Furthermore, the reciprocal of the smallest 
singular values in Eq. (18) greatly amplifies the measurement noise in calculating the load. An appropriate option to overcome this problem is to select a filter function $W_{\varphi}\left(\sigma_{i}^{2}\right)$ to multiply the reciprocal of singular values. With the help of filter function, the expression $W_{\varphi}\left(\sigma_{i}^{2}\right) \sigma_{i}^{-1}$ approaches zero as $\sigma_{i}$ decays gradually to zero. The influence of noise amplification is thereby controlled through the filter function [43].

$$
\mathbf{F}_{\varphi}=\mathbf{F}+\sum_{i=1}^{m} W_{\varphi}\left(\sigma_{i}^{2}\right) \sigma_{i}^{-1}\left(\mathbf{u}_{i}^{\mathrm{T}} \cdot \eta_{i}\right) \mathbf{v}_{i}
$$

where $\varphi$ refers to a constant regularization parameter, and $\mathbf{F}_{\varphi}$ refers to the load computed from a regularized measured noisy response.

A proper regularized filter as well as a step filter namely TSVD filter is here briefed. In TSVD, the function $W_{\varphi}\left(\sigma_{i}^{2}\right)$ can only take value of 0 or 1 ,

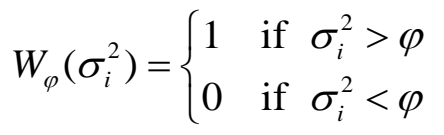

Base on this filter, the TSVD-based scheme formulation is given as follows:

$$
\mathbf{F}_{\varphi}=\mathbf{F}+\sum_{i: \sigma_{i}^{2}>\varphi} \sigma_{i}^{-1}\left(\mathbf{w}_{i}^{\mathrm{T}} \cdot \eta_{i}\right) \mathbf{v}_{i}
$$

To solve Eq. (21), the critical value of $\varphi$ in the regularized filter is a very important parameter. It is noted that when the value of $\varphi$ is too large, more singular values are filtered, which leads to over regularization in the identification process. This results in the over-smoothing for the identified load, in other word, there is insufficient data to fit $\eta_{i}$, i.e. the residual norm $\left\|\mathbf{G F}_{\varphi}-\mathbf{e r r}\right\|$ will be too large. On the other hand, when the value of $\varphi$ is too small, the accuracy of the residual norm can be ensured. However, more singular 
values are induced with small $\varphi$, and the regularized solution of $\left\|\mathbf{F}_{\varphi}\right\|$ tends to be large or oscillating. The L-curve criterion [43], which can provide a guideline to balance the residual norm and the regularized solution, is thus applied to properly select the optimal regularization parameter $\varphi$. Actually, both the regularized solution and the residual norm are the function of regularization parameter $\varphi$, and these two relationships form an optimum solution of two objective functions. It is suggested that the corner of the L-shaped curve will give the best regularization parameter. More details can be found in [43].

Once Eq. (21) is solved, we can identify the force acting on the structure using the acoustic pressure. In fact, load identification can also be implemented by Eq. (21) when the structure response is obtained. The advantage of the non-contact acoustic pressure is that it can provide much more convenience.

\section{Non-probabilistic methods for the uncertain structure}

According to Eqs. (19) and (21), we can see that load identification is based on the deterministic ASI system. However, a complicated structural-acoustic system often contains many uncertain factors, such as the errors of manufacture, the model inaccuracies, the unpredictable loading conditions, and the aggressive environment factors. Hence, the sound pressure generated from the structural-acoustic system is always subjected to these uncertain parameters. Without loss of generality, in this work, it is assumed that the material properties of the structure in the coupled ASI system are considered as the 
uncertain factors.

Generally, the probability model and fuzzy-set model can well transform the uncertain parameters with their precise information (i.e. the probability density function and the fuzzy membership functions). Nevertheless, constructing the precise distribution functions of the uncertain parameters is challenging because a lot of samples are required to obtain the priori statistics information, which is of great burden for the practical engineering. In this case, a realistic model namely the non-probabilistic model with only knowing the extreme bounds rather than the sufficient statistical information is more preferred to tackle with the uncertain parameters. In this section below, two kinds of non-probabilistic model including ellipsoid and interval models are utilized to transform the uncertain parameters.

\subsection{Ellipsoid model method for load identification with uncertain parameters}

The bounded convex set-ellipsoid which describes the $m$ uncertain parameters for the problem is defined as follows:

$$
Z(l, e)=\left\{\lambda: \sum_{i=1}^{m} \frac{\left(\lambda_{i}-\lambda_{i}^{0}\right)^{2}}{e_{i}^{2}} \leq l^{2}\right\}
$$

where $l$ is a positive constant which measures the size of the ellipsoid, it is also called the radius of the ellipsoid; $e=\left(e_{i}\right)_{m}$ is the semi-axes of the ellipsoid; $\lambda^{0}=\left(\lambda_{i}^{0}\right)_{m}$ is the central point of the ellipsoid and it is chosen as the nominal value; $Z$ is the convex ellipsoid domain formed from $m$ uncertain variables. A bi-dimensional ellipsoid set can be described in the sketch map as shown in Fig. 1: 


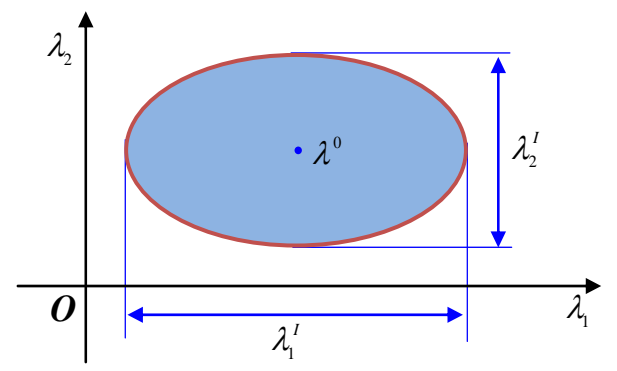

Fig. 1 A bi-dimensional ellipsoidal model

Following the idea of convex ellipsoid models, it is assumed that the $m$-dimensional vector $\lambda=\left(\lambda_{i}\right)_{m}$ is used to denote the uncertain structural parameters existing in structural property.

$$
\lambda_{i}=\lambda_{i}^{0}+\delta_{i}, i=1,2, \ldots, m
$$

where $\delta=\left(\delta_{i}\right)_{m}$ are the small variation of the uncertain parameters. It should be pointed that since the system is a causal, linear and time-invariant system, the load can be described as the function of the uncertain parameter which is expressed as $\tilde{F}=F(\lambda)$. In other words, the load to be identified is also an uncertain function that can be approximated by employing the Taylor series expansion with respect to the ellipsoid uncertain parameters.

$$
F(\lambda)=F\left(\lambda^{0}+\delta\right)=F\left(\lambda^{0}\right)+\sum_{i=1}^{m} \frac{\partial F\left(\lambda^{0}\right)}{\partial \lambda_{i}} \delta_{i}+\frac{1}{2 !} \sum_{i=1}^{m} \frac{\partial^{2} F\left(\lambda^{0}\right)}{\partial \lambda_{i}^{2}} \delta_{i}+\ldots
$$

where $\partial F\left(\lambda^{0}\right) / \partial \lambda_{i}$ represents the first order partial derivative of the mean load with respect to the $i$-th uncertain parameter. As mentioned by several authors, [21, 37], the improvement in accuracy obtained by using the higher order perturbation terms is rather small compared with the increase of the computational complexity and the computational 
burden. An approximation using the first-order Taylor expansion with simultaneously neglecting the higher order perturbation terms for the linear function is feasible without suffering a considerable computational burden, thus we can arrive at:

$$
F(\lambda)=F\left(\lambda^{0}+\delta\right)=F\left(\lambda^{0}\right)+\sum_{i=1}^{m} \frac{\partial F\left(\lambda^{0}\right)}{\partial \lambda_{i}} \delta_{i}
$$

The sensitivity notation in the right hand of Eq. (25) can be defined as:

$$
s=\left(\frac{\partial F\left(\lambda^{0}\right)}{\partial \lambda_{1}}, \frac{\partial F\left(\lambda^{0}\right)}{\partial \lambda_{2}}, \cdots, \quad \frac{\partial F\left(\lambda^{0}\right)}{\partial \lambda_{m}}\right)^{\mathrm{T}}
$$

Thus Eq. (25) can be expressed as:

$$
F(\lambda)=F\left(\lambda^{0}+\delta\right)=F\left(\lambda^{0}\right)+s^{\mathrm{T}} \delta
$$

Eq. (27) forms the set of the load to be identified. Generally, its bounds namely the extreme values are determined through [44]:

$$
F_{\text {ext }}=\underset{\lambda \in Z(l, e)}{\operatorname{extremum}}\left\{F\left(\lambda^{0}\right)+s^{\mathrm{T}} \delta\right\}
$$

where $F_{\text {ext }}$ is the extreme bounds of the ellipsoid model. Based on the ellipsoid set theory, the bounds of the load can be taken only when the uncertain parameters apply the bound value of $Z(l, e)$, i.e. on the ellipsoid shell

$$
\mathrm{B}(l, e)=\left\{\lambda: \sum_{i=1}^{m} \frac{\left(\lambda_{i}-\lambda_{i}^{0}\right)^{2}}{e_{i}^{2}}=l^{2}\right\}
$$

where $\mathrm{B}(l, e)$ denotes the boundary of the ellipsoid set. By applying the boundary condition of the uncertain parameters, Eq. (29) can be taken its extreme value and rewritten as follows [44]:

$$
F_{\text {ext }}=\underset{\lambda \in B(l, e)}{\operatorname{extremum}}\left\{F\left(\lambda^{0}\right)+s^{\mathrm{T}} \delta\right\}
$$


A lot of methods can be employed to obtain the boundary of the load such as the Monte-Carlo method and the interval method. In this paper, the ellipsoid method is served as the approach to obtain the bounds. Given a $m \times m$ dimensions diagonal matrix $\psi=\operatorname{diag}\left\{1 / e_{i}^{2}\right\}$, it denotes the characteristic matrix of the ellipsoid set, where $e_{i}$ is the $i$-th semimajor axis of the uncertain domain and determines the direction of the $i$-th parameter. The boundary condition described by Eq. (29) becomes:

$$
\delta^{\mathrm{T}} \psi \delta-l^{2}=0
$$

The load identification of the ASI system with uncertain structural parameters can be transformed into an extremal value problem. This case is equivalent to solving Eq. (30) subject to the constraints condition Eq. (31). The Lagrange multiplier method is used to further solve the extreme value by using the Lagrange function:

$$
L(\delta)=s^{T} \delta+\alpha\left(\delta^{\mathrm{T}} \psi \delta-l^{2}\right)
$$

where $\alpha$ represents the Lagrange multiplier. When the load takes its extreme value, the following necessary condition of the derivatives should be satisfied:

$$
\frac{\partial L}{\partial \delta}=s^{T}+2 \alpha \psi \delta=0
$$

Arranging the above equation yields:

$$
\delta=-\frac{1}{2 \alpha} \psi^{-1} s
$$

With the expression of Eqs. (31) and (33), the following form can be deduced:

$$
\alpha= \pm \frac{1}{2 l} \sqrt{s^{\mathrm{T}} \psi^{-1} s}
$$

Submitting Eq. (35) into Eq. (34), the perturbation in Eq. (30) can be obtained: 


$$
\delta= \pm \frac{l}{\sqrt{s^{\mathrm{T}} \psi^{-1} s}} \psi^{-1} s
$$

Finally, the extreme value of the load to be identified can be rearranged as:

$$
\begin{gathered}
F_{\max }=F\left(\lambda^{0}\right)+l \sqrt{s^{\mathrm{T}} \psi^{-1} s}=F^{0}+l \sqrt{\sum_{i=1}^{m}\left(e_{i} \frac{\partial F\left(\lambda^{0}\right)}{\partial \lambda_{i}}\right)^{2}} \\
F_{\min }=F\left(\lambda^{0}\right)-l \sqrt{s^{\mathrm{T}} \psi^{-1} s}=F^{0}-l \sqrt{\sum_{i=1}^{m}\left(e_{i} \frac{\partial F\left(\lambda^{0}\right)}{\partial \lambda_{i}}\right)^{2}}
\end{gathered}
$$

where $F^{0}$ is the central point load of the ellipsoid, and the first partial derivatives $\partial F\left(\lambda^{0}\right) / \partial \lambda_{i}$ stands for the sensitivity of the center point load with respect to the uncertain parameter when it perturbs at the center of the ellipsoid. By introducing the convex model analysis, the maximum and minimum ranges of the load in Eqs. (37)-(38) are transformed into the computation of the central point load and the sensitivity analysis. The central point load is determined through the methods introduced in Section 2, and the sensitivity analysis is addressed in Section 3.3.

\subsection{Interval model for inverse problem with uncertain parameters}

In interval mathematics, the uncertain parameters can be described by an interval vector. In order to make a comparison with convex model, the upper and lower bounds of the uncertain parameters are assumed to equal to the semi-axes of the ellipsoid respectively. The relationship can be depicted as Eq. (39)

$$
\lambda^{I}=[\underline{\lambda}, \bar{\lambda}]=\left\{\lambda: \underline{\lambda} \leq \lambda \leq \bar{\lambda}, \lambda=\left(\lambda_{i}\right)_{m}, \underline{\lambda}=\left(\underline{\lambda}_{i}\right)_{m}, \bar{\lambda}=\left(\bar{\lambda}_{i}\right)_{m} \in R^{m}\right\}
$$

where $\underline{\lambda}$ and $\bar{\lambda}$ are the left and right bounds of the interval respectively. This relationship can be drawn in Fig. 2 through a bi-dimensional problem. 


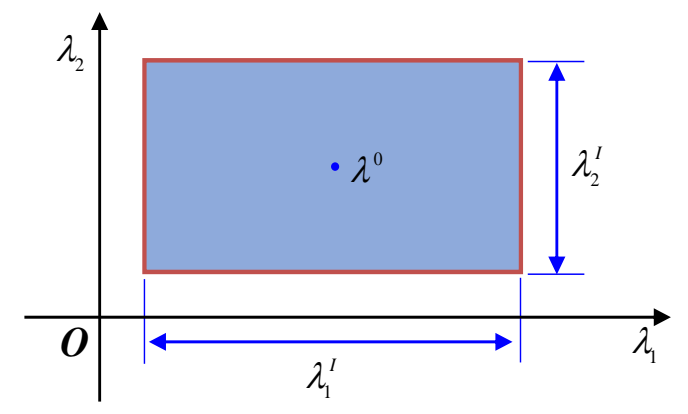

Fig. 2 A bi-dimensional interval model

In Fig. 2 the rectangle stands for the interval model, and $\lambda^{0}$ is the center point and can be yielded through:

$$
\lambda^{0}=\frac{\underline{\lambda}+\bar{\lambda}}{2}, \lambda^{r}=\frac{\bar{\lambda}-\underline{\lambda}}{2}
$$

where $\lambda^{r}$ is the radius of an interval vector. Since the uncertain parameter can be expressed through the interval model and are expressed in Eq. (39), the interval vector can be rewritten in terms of $\lambda^{0}$ and $\lambda^{r}$ as follows:

$$
\lambda^{I}=[\underline{\lambda}, \bar{\lambda}]=\left[\lambda^{0}-\lambda^{r}, \lambda^{0}+\lambda^{r}\right]=\lambda^{0}+[-1,1] \lambda^{r}=\lambda^{0}+\xi \lambda^{r}
$$

where $\xi=[-1,1]$ is the uncertain parameter in the interval vector denoting the interval uncertainty. Similar to Eq. (25), the load bounds of the identified load can be simply computed with the first-order Taylor expansion:

$$
\begin{aligned}
& \bar{F}=F^{0}+\sum_{i=1}^{m}\left|\frac{\partial F\left(\lambda^{0}\right)}{\partial \lambda_{i}}\right| \cdot \xi_{i} \\
& \underline{F}=F^{0}-\sum_{i=1}^{m}\left|\frac{\partial F\left(\lambda^{0}\right)}{\partial \lambda_{i}}\right| \cdot \xi_{i}
\end{aligned}
$$

where $\bar{F}$ and $\underline{F}$ are the upper and lower bounds of the force to be identified for the 
interval model, and $\xi_{i}$ is the $i$-th interval uncertainty. The sensitivities can be figured out via the same way as the convex ellipsoid model.

\subsection{Sensitivity analysis of the load with respect to the uncertain parameters}

Since the structural parameter is considered to have the small uncertainty, the center point load in Eqs. (37)-(38) and (42)-(43) is calculated through the inverse analysis method introduced in Section 2. The sensitivity analysis of the dynamic load with respect to the parameter is computed through the same way as finite element method. Given that one of the structural nodes is subjected to an arbitrary pulse force $\mathbf{F}_{\mathrm{s}}$, where $\mathbf{F}_{\mathrm{s}}=\{0,0, \ldots, F(t), \ldots\}^{\mathrm{T}}$, the dynamic Eq. (1) for the ASI system with structural uncertain parameters can be expressed as [2]:

$$
\mathbf{M}_{\mathrm{s}}(\lambda) \ddot{\mathbf{u}}(\lambda, t)+\mathrm{C}_{\mathrm{s}}(\lambda) \dot{\mathbf{u}}(\lambda, t)+\mathbf{K}_{\mathrm{s}}(\lambda) \mathbf{u}(\lambda, t)=\mathbf{F}_{\mathrm{s}}
$$

where $\mathbf{M}_{\mathrm{s}}(\lambda), \mathbf{C}_{\mathrm{s}}(\lambda)$ and $\mathbf{K}_{\mathrm{s}}(\lambda)$ are the uncertain mass, damping, and stiffness matrices, respectively. $\mathbf{u}(\lambda, t)$ is the nodal displacement vector. The sensitivity analysis with respect to the central point value $\lambda^{0}$ can be obtained by applying the first-order partial derivatives to Eq. (44)

$$
\mathbf{M}_{\mathrm{s}}\left(\lambda^{0}\right) \frac{\partial \ddot{\mathbf{u}}\left(\lambda^{0}, t\right)}{\partial \lambda_{i}^{0}}+\mathbf{C}_{\mathrm{s}}\left(\lambda^{0}\right) \frac{\partial \dot{\mathbf{u}}\left(\lambda^{0}, t\right)}{\partial \lambda_{i}^{0}}+\mathbf{K}_{\mathrm{s}}\left(\lambda^{0}\right) \frac{\partial \mathbf{u}\left(\lambda^{0}, t\right)}{\partial \lambda_{i}^{0}}=\mathbf{F}\left(\lambda^{0}, t\right)
$$

where $\mathbf{F}\left(\lambda^{0}, t\right)=\partial \mathbf{F}_{\mathrm{s}} / \partial \lambda^{0}$ is the explicit form of the force sensitivity with respect to the uncertain parameters, it can be finally yielded by: 


$$
\mathbf{F}\left(\lambda^{0}, t\right)=\frac{\partial \mathbf{F}\left(\lambda^{0}\right)}{\partial \lambda^{0}}=-\ddot{\mathbf{u}}\left(\lambda^{0}, t\right) \frac{\partial \mathbf{M}_{\mathrm{s}}\left(\lambda^{0}\right)}{\partial \lambda_{i}^{0}}-\dot{\mathbf{u}}\left(\lambda^{0}, t\right) \frac{\partial \mathbf{C}_{\mathrm{s}}\left(\lambda^{0}\right)}{\partial \lambda_{i}^{0}}-\mathbf{u}\left(\lambda^{0}, t\right) \frac{\partial \mathbf{K}_{\mathrm{s}}\left(\lambda^{0}\right)}{\partial \lambda_{i}^{0}}
$$

Following the assemble methods of the stiff and mass matrices in the FEM, the first-order partial derivatives of the global mass, damping and stiffness matrices with respect to the parameters can be assembled as follows:

$$
\begin{gathered}
\frac{\partial \mathbf{M}_{\mathrm{s}}\left(\lambda^{0}\right)}{\partial \lambda_{i}^{0}}=\frac{\partial}{\partial \lambda_{i}} \sum_{j=1}^{M \text { cell }} \mathbf{M}_{\mathrm{s}, j}\left(\lambda^{0}\right)=\sum_{j=1}^{\text {Mcell }} \frac{\partial \mathbf{M}_{\mathrm{s}, j}\left(\lambda^{0}\right)}{\partial \lambda_{i}}=\sum_{j=1}^{M \text { cell }} \frac{\partial}{\partial \lambda_{i}} \int_{\Omega_{\mathrm{s}}} \rho_{\mathrm{s}} \mathbf{N}_{\mathrm{s}}^{\mathrm{T}} \mathbf{N}_{\mathrm{s}} \mathrm{d} \Omega \\
\frac{\partial \mathbf{C}_{\mathrm{s}}\left(\lambda^{0}\right)}{\partial \lambda_{i}^{0}}=\frac{\partial}{\partial \lambda_{i}} \sum_{j=1}^{M \text { cell }} \mathbf{C}_{\mathrm{s}, j}\left(\lambda^{0}\right)=\sum_{j=1}^{M \text { cell }} \frac{\partial \mathbf{C}_{\mathrm{s}, j}\left(\lambda^{0}\right)}{\partial \lambda_{i}}=\sum_{j=1}^{M c e l l} \frac{\partial}{\partial \lambda_{i}} \int_{\Gamma_{\mathrm{s}}} \mathbf{N}_{\mathrm{s}}^{\mathrm{T}} \mathbf{N}_{\mathrm{s}} \varsigma \mathrm{d} \Gamma \\
\frac{\partial \mathbf{K}_{\mathrm{s}}\left(\lambda^{0}\right)}{\partial \lambda_{i}^{0}}=\frac{\partial}{\partial \lambda_{i}} \sum_{j=1}^{M \text { cell }} \mathbf{K}_{\mathrm{s}, j}\left(\lambda^{0}\right)=\sum_{j=1}^{M c e l l} \frac{\partial}{\partial \lambda_{i}} \int_{\Omega_{\mathrm{s}}} \mathbf{B}_{\mathrm{s}}^{\mathrm{T}} \mathbf{D}_{\mathrm{s}} \mathbf{B}_{\mathrm{s}} \mathrm{d} \Omega
\end{gathered}
$$

where $i$ is the number of the uncertain parameters, Mcell is the number of the element in the global structure region; $\partial \mathbf{M}_{\mathrm{s}, j}\left(\lambda^{0}\right) / \partial \lambda_{i}$ is the first order partial derivative of the structural mass matrix with respect to the parameter $\lambda_{i}$ for the $j$-th element; the notation $\sum_{j=1}^{\text {Mcell }}$ means the matrix is assembled in a finite element approach rather than a simple sum operation. For example, the structure density is the first uncertain parameter in the ASI system, and the sensitivity of the structural mass matrix with respect to the structure density can be computed as follows:

$$
\frac{\partial \mathbf{M}_{\mathrm{s}}\left(\rho_{\mathrm{s}}^{0}\right)}{\partial \rho_{\mathrm{s}}}=\frac{\partial}{\partial \rho_{\mathrm{s}}} \sum_{j=1}^{\text {Mcell }} \mathbf{M}_{\mathrm{s}, 1}\left(\rho_{\mathrm{s}}^{0}\right)=\sum_{j=1}^{\text {Mcell }} \frac{\partial \mathbf{M}_{\mathrm{s}, 1}\left(\rho_{\mathrm{s}}^{0}\right)}{\partial \rho_{\mathrm{s}}}=\sum_{j=1}^{M \text { cell }} \int_{\Omega_{\mathrm{s}}} \mathbf{N}_{\mathrm{s}}^{\mathrm{T}} \mathbf{N}_{\mathrm{s}} \mathrm{d} \Omega
$$

The derivatives of the mass and stiffness matrices with respect to the corresponding uncertain parameters can be computed as the assembling way of Eq. (50). Once the 
derivatives in Eqs. (47)-(50) are obtained, the sensitivity analysis of the load with respect to the uncertain parameters is herein solved. Further, the load bounds computed through Eqs. (37-38) and (42-43) are determined.

To sum up, the flowchart of the implementation of the acoustic pressure-based methods in the ASI system involving non-probabilistic uncertainties is shown in Fig. 3:

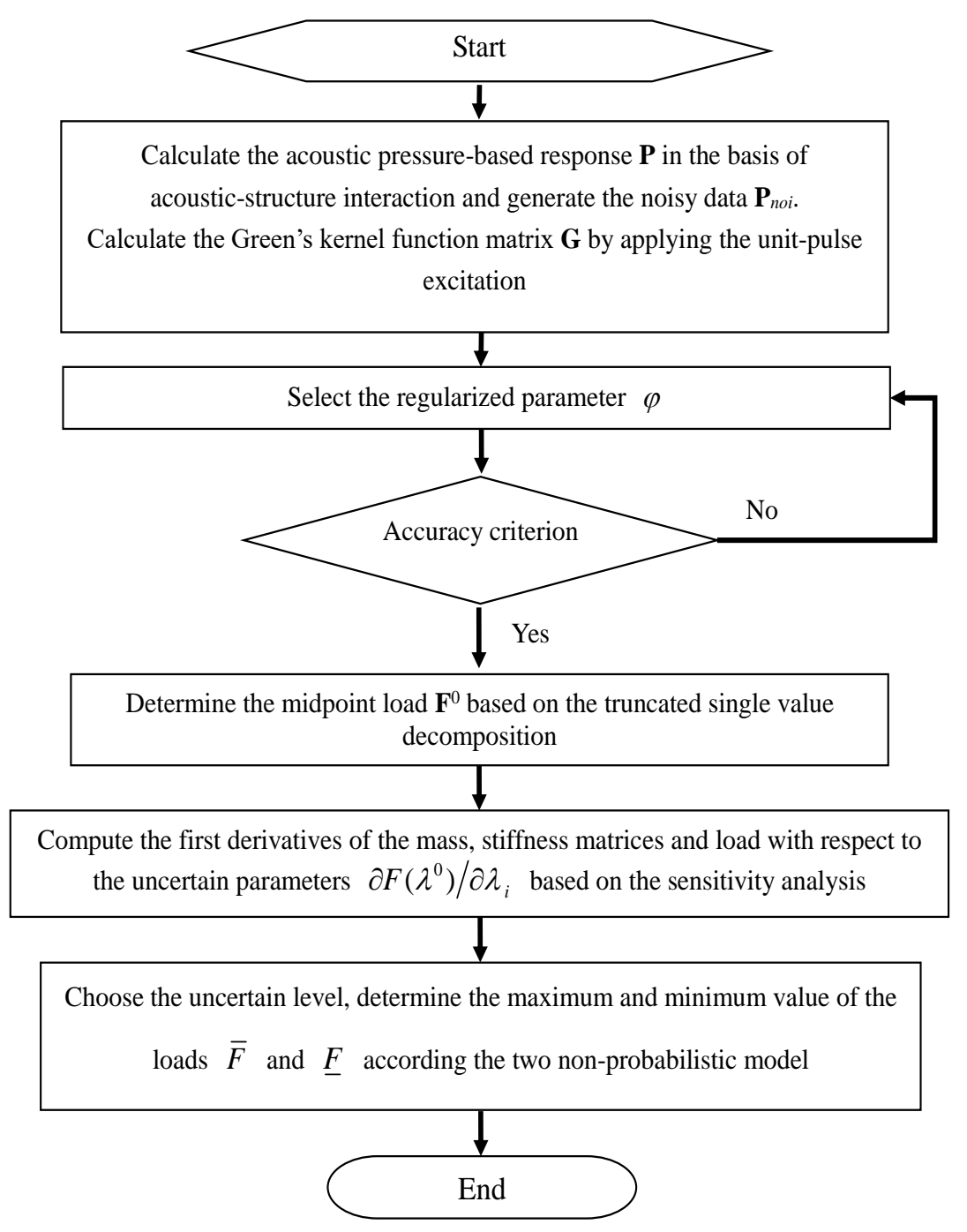

Fig. 3 The flowchart of the implementation of the proposed method 


\section{Numerical examples}

In this section, three numerical examples are investigated to validate the effectiveness of the proposed acoustic pressure-based method in the ASI system with non-probabilistic uncertain variables. A square plate coupled with the cubic acoustic cavity is firstly studied to illustrate how to identify different concentrated loads history through the proposed pressure-based method. Next, the non-probabilistic uncertainty of the ASI system is taken into consideration in the second example, where a circular plate coupled with cylindrical cavity is investigated. In the third example, a more practical model of vehicle roof coupled with passenger cavity with complicated geometry is studied. In this work, we concentrate the work in identifying the load history and quantifying the influence of the uncertain factors, while the identification of the load location is another study aspect, which can be found in references [7, 42].

\subsection{A square plate coupled with the cubic cavity}

The square plate coupled with the cubic acoustic cavity that owns $500 \mathrm{~mm}$ length in three-dimensional as shown in Fig. 4 is firstly investigated. The thickness of the square plate is $1 \mathrm{~mm}$. The Young's modulus of the structural material is $E=210 \mathrm{GPa}$, the density is $\rho_{\mathrm{s}}=7,800 \mathrm{~kg} / \mathrm{m}^{3}$, the Poisson's ratio is $v=0.3$, and the structural damping factor is set as $\zeta=0.06$. The properties of the acoustic cavity expressed by the acoustic velocity and mass density are $c=340 \mathrm{~m} / \mathrm{s}$ and $\rho_{\mathrm{a}}=1.225 \mathrm{~kg} / \mathrm{m}^{3}$ respectively. The sides surrounding the square plate are fixed, and the top wall of the cubic cavity is coupled with 
the vibration of the structure. In addition, it is assumed that the other walls of the cavity are completely rigid. In the preprocessing, the square plate and the cubic cavity are discretized by 400 quadrilateral elements and 23,175 tetrahedral elements, respectively; the whole domain contains 5,175 nodes.

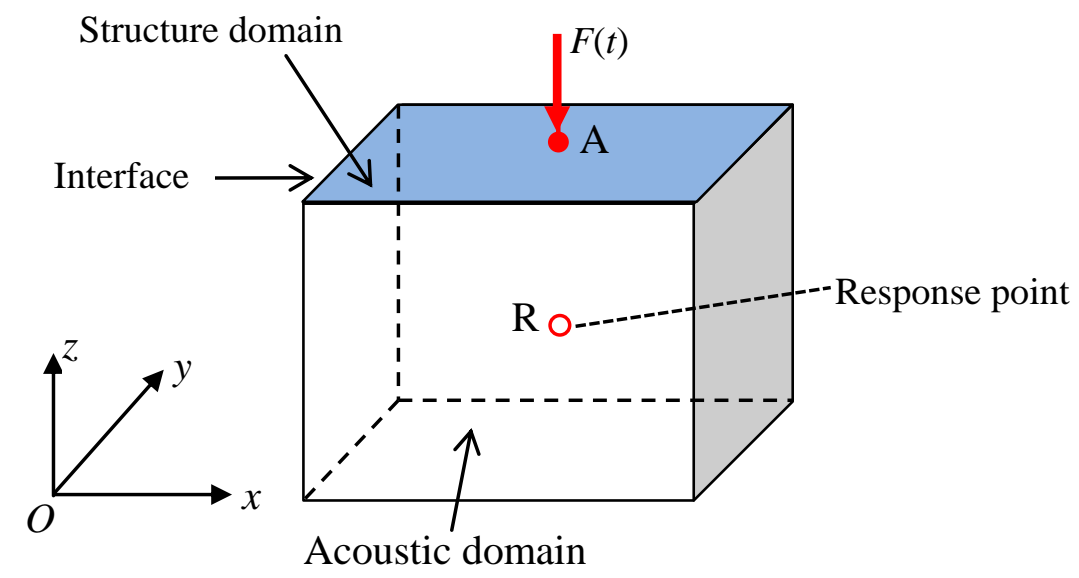

Fig. 4 The cubic coupled model

Here, four cases of loads (as shown in Table 1) are loaded on the central point (marked as A) of the plate, and the geometric center (marked as R) of the cubic is selected as the response point, as shown in Fig. 4. Four cases of the excitation forces which differ from frequency components (as listed in Table 1) are taken into investigation. Case 1 has the single frequency characteristic, case 2 has two single frequencies, case 3 is the impulse force, and case 4 has the variable frequency characteristic.

Table 1: Four different cases of loads

$\begin{array}{ccl}\text { Case 1 } & F=50 \sin (80 \pi t) & \text { Frequency } f=40 \mathrm{~Hz} \\ \text { Case 2 } & F=50[\sin (200 \pi t)+\sin (80 \pi t)] & \text { Contains two frequency components } \\ \text { Case 3 } & F=50 \text { at } t=0.05 \mathrm{~s} & \text { Impulse force at } t=0.05 \mathrm{~s}\end{array}$


Generally, the noise is directly added to the computer-generated response to simulate the measured acoustic pressure response, here we use the popular noisy model which is defined as follows $[9,10]$ :

$$
p_{\text {noi }}=p_{c a l}+l_{\text {noi }} \cdot \operatorname{std}\left(p_{c a l}\right) \cdot \operatorname{rand}(-1,1)
$$

where $p_{n o i}$ is the polluted pressure signal, $p_{c a l}$ is the computer-generated signal, $\operatorname{std}\left(p_{c a l}\right)$ is the standard deviation of $p_{c a l}$, $\operatorname{rand}(-1,1)$ represents the random number between -1 and 1 , and $l_{\text {noi }}$ represents the polluted level of the noisy response. Two noise levels, namely $5 \%$ and $10 \%$ are studied. The identified results for the four cases of load through the proposed pressure-based method using the TSVD regularization approach with L-curve criterion are illustrated in Figs. 5-8.

As shown in Figs. 5-8, when the noise takes the two kinds of levels, namely 5\% and $10 \%$, the reconstructed results for different types of force are all in an excellent agreement with the exact solution by using the TSVD regularized method. Interestingly, the proposed acoustic pressure-based method is also found to be effective to identify the impact force as outlined in Fig. 7. More importantly, the proposed novel sound pressure method is a non-contact measurement, which is more convenient in the load identification process compared with the conventional structural responses processed in a contact way. It should be pointed that the error between the identified load and the exact load becomes large near the end of the time period. Two factors may be responsible for this. The first one is that the 
random phases may lead to the phase-shift phenomena with the introduction of noise[45], as part of noise may intermingle with the actual dynamic load. Consequently, the effects of this noise cannot be completely regularized and discarded. The second factor is that the regularized norm is oscillating in order to reduce the residual norm near the end of the time period, since the responses are oscillating when the load is no longer applied.

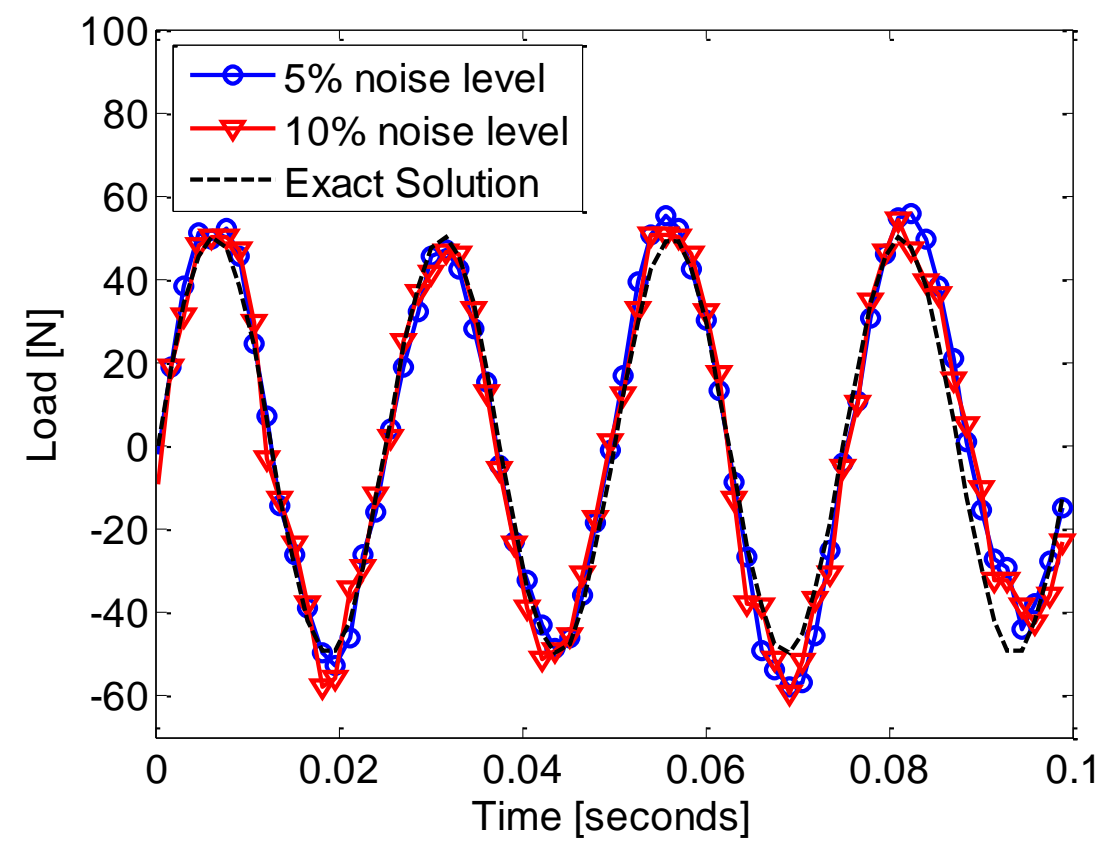

Fig. 5. The identified result of Case 1 (Frequency $f=40 \mathrm{~Hz}$ ) with $5 \%$ and $10 \%$ noise levels 


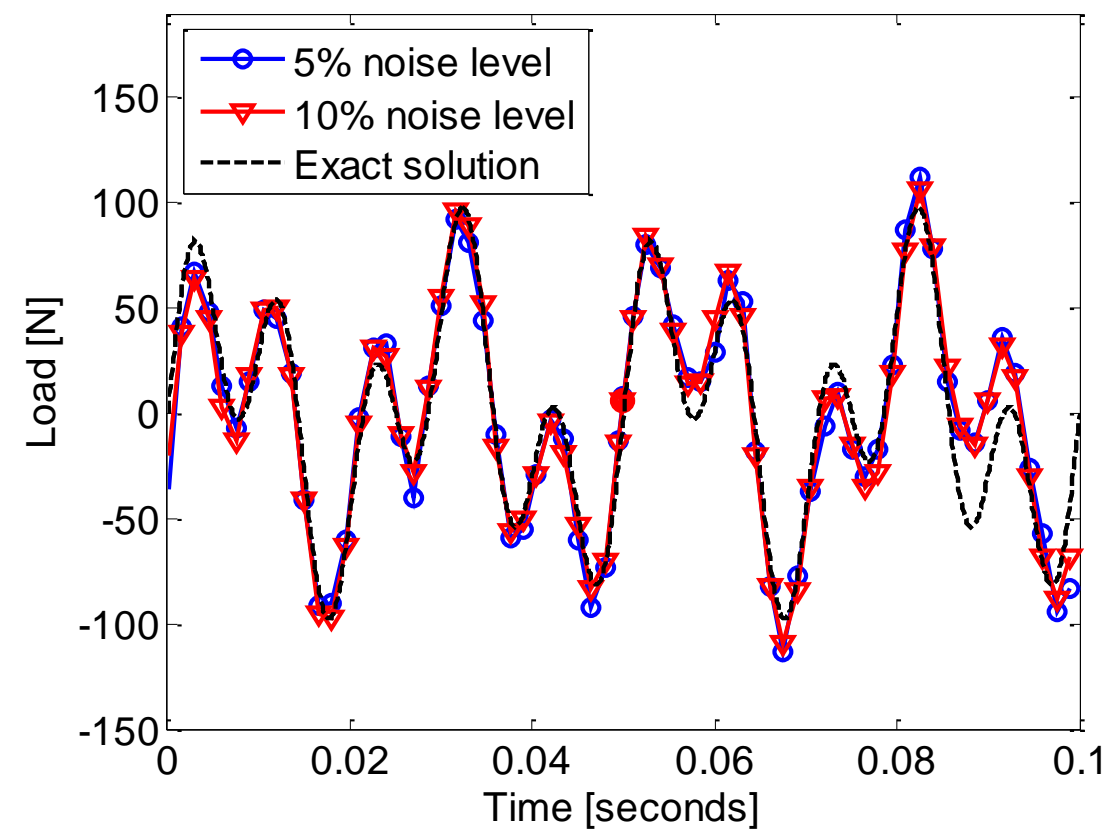

Fig. 6 The identified result of case 2 (Contain two frequency components) with 5\% and 10\% noise levels

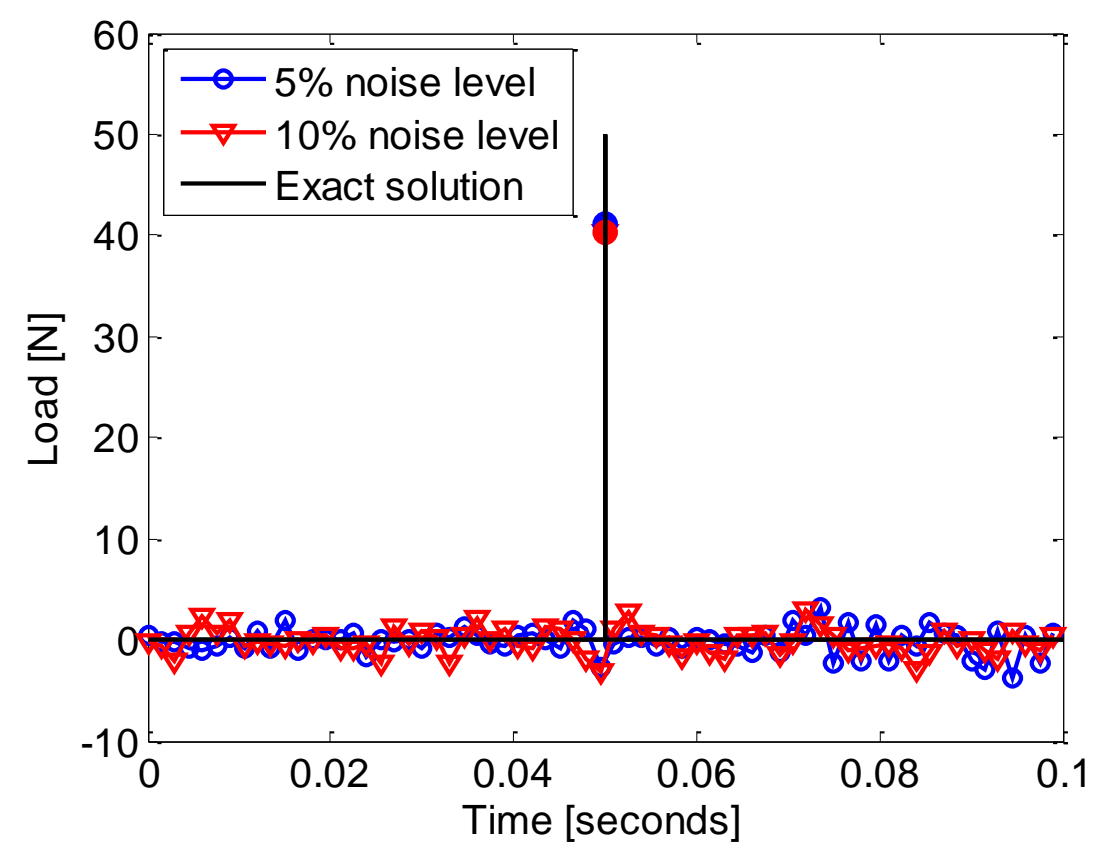

Fig. 7 The identified result of case 3 (Impulse force at $t=0.05 \mathrm{~s}$ ) with $5 \%$ and $10 \%$ noise levels 


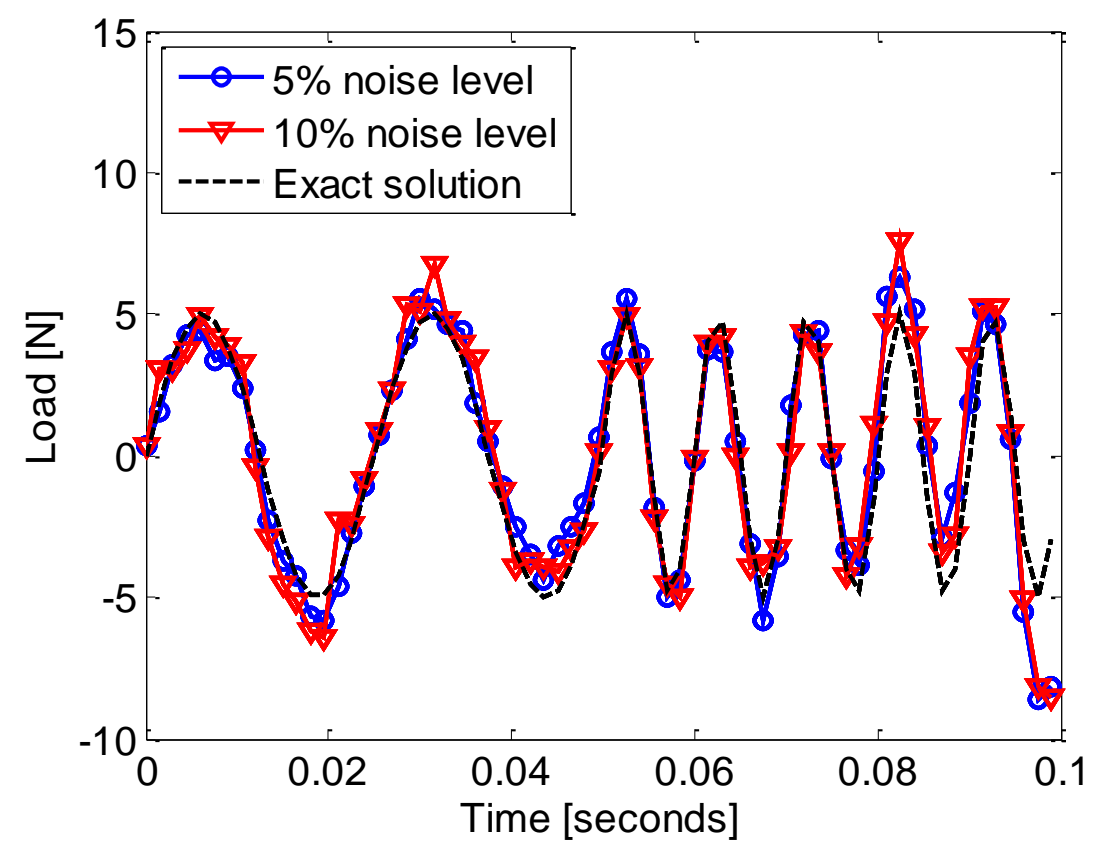

Fig. 8 The identified result of case 4 (Force with piecewise function) with 5\% and 10\% noise levels

\subsection{A circular plate coupled with the cylindrical cavity}

In this example, a circular-plate coupled with the cylindrical cavity, as demonstrated in Fig. 9 is studied. The radius of the cylinder is $250 \mathrm{~mm}$, the height in $z$ direction is 500 $\mathrm{mm}$, and the thickness of the circular plate is $1 \mathrm{~mm}$. In the preprocessing, the plate and the cylindrical cavity are discretized by 488 quadrilateral elements and 134,486 tetrahedral elements, respectively. The structural parameters of the plate are: Young's modulus $E^{0}=210 \mathrm{GPa}$, Poisson's ratio $v=0.3$, density $\rho_{\mathrm{s}}^{0}=7,800 \mathrm{~kg} / \mathrm{m}^{3}$, and the structural damping factor $\zeta=0.06$. The property of the acoustic part expressed by the sound velocity and mass density are $c=340 \mathrm{~m} / \mathrm{s}$ and $\rho_{a}=1.225 \mathrm{~kg} / \mathrm{m}^{3}$, respectively. In this numerical example, without loss of generality, the Young's modulus and the density of the structure are treated as the non-probabilistic parameters; the thickness of shell, the density 
and the sound speed of air are treated as the constants. These parameters are assumed to be independent of each other.

$$
\frac{\left(E-E^{0}\right)^{2}}{\left(e E^{0}\right)^{2}}+\frac{\left(\rho_{\mathrm{s}}-\rho_{\mathrm{s}}^{0}\right)^{2}}{\left(e \rho_{\mathrm{s}}^{0}\right)^{2}} \leq 1
$$

where $e$ is a factor describing the uncertainty level of the uncertain parameters, and its variation range is $(0,1)$. Two uncertainty levels are taken into consideration i.e. 1) $e=0.05$, and 2) $e=0.1$.

The sides surrounding the circular plate are fixed. A vertical load has the form of $F=20 \sin (100 \pi t)$ is acting on the central point (marked as A in Fig. 9) of the plate. The top surface of the cavity is subjected to the vibration coming from the structure, and the cylindrical and bottom surface of the cavity are considered completely rigid.

Table 2: Characters of the uncertain parameters depicted in two models

\begin{tabular}{cccc}
\hline Uncertainty level & Interval parameters & \multicolumn{2}{c}{ Ellipsoid parameters } \\
\hline $5 \%$ & $E \in[199.5,220.5] \mathrm{GPa}$ & $E^{0}=210 \mathrm{GPa}$ & \\
& $\rho \in[7410,8190] \mathrm{kg} / \mathrm{m}^{3}$ & $\rho_{s}^{0}=7800 \mathrm{~kg} / \mathrm{m}^{3}$ & \\
& $E \in[189,231] \mathrm{GPa}$ & $E^{0}=210 \mathrm{GPa}$ & \\
$10 \%$ & $\rho \in[7020,8580] \mathrm{kg} / \mathrm{m}^{3}$ & $\rho_{s}^{0}=7800 \mathrm{~kg} / \mathrm{m}^{3}$ & $e=0.1$ \\
\hline
\end{tabular}

As the pressure-based method with TSVD regularized approach has shown its good effect to identify the load in the first example, it will be still employed to identify the central point load in this example. Similarly, the noise model applied in the first application is continuously adapted to this cylinder model. When the two kinds of noise levels are directly added to computer-generated acoustic pressure response, the acoustic 
response of point $\mathrm{E}$ is obtained and plotted in Fig. 10. As the uncertain parameter takes the mean value, the central point load can be identified by using the TSVD method based on acoustic pressure, and the results are shown in Fig. 11.

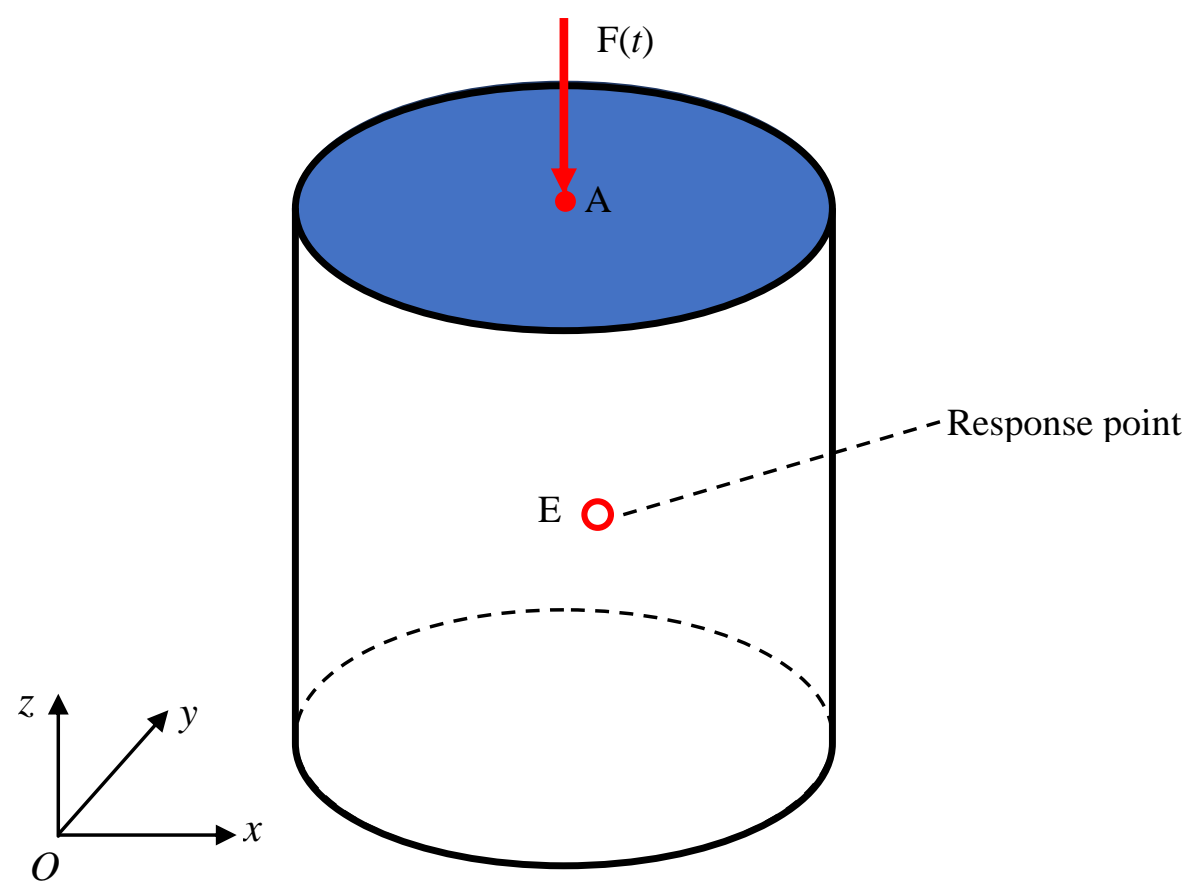

Fig. 9 The 3D model of the simple-plate coupled with the cylindrical cavity

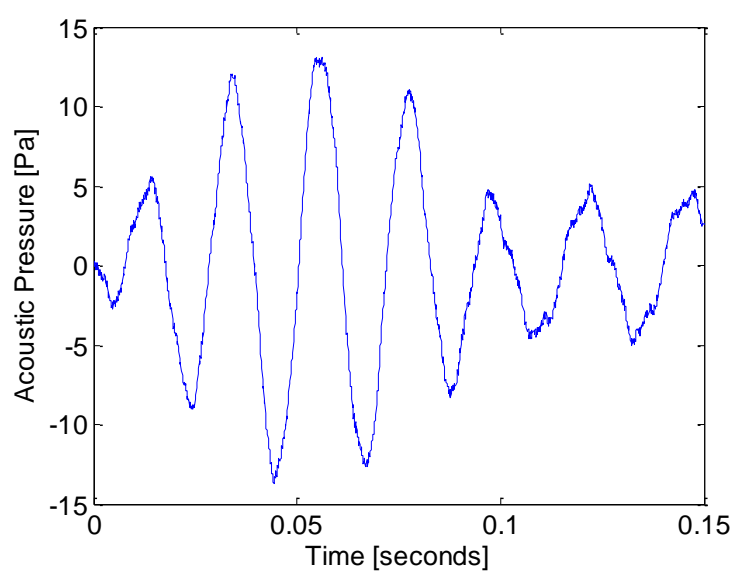

(a)

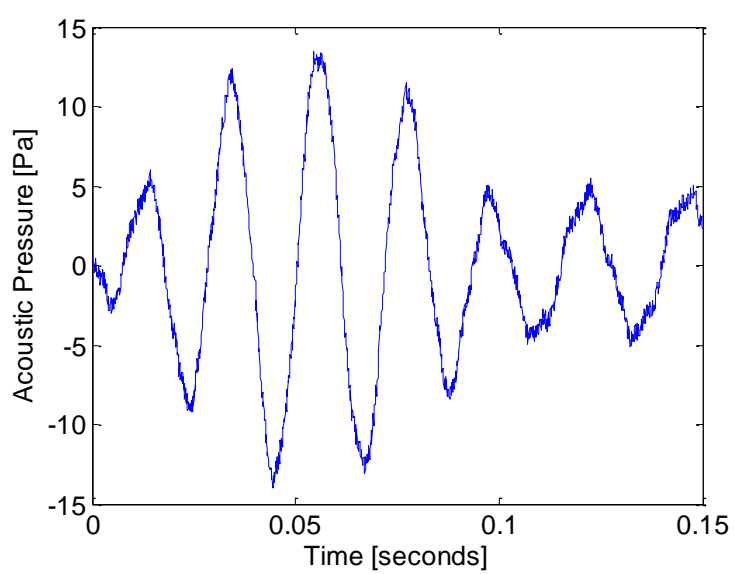

(b)

Fig. 10 The noisy acoustic pressure response of point E. (a) 5\% noise level, (b) $10 \%$ noise level It can be seen from Fig. 11 that the central point load can be identified stably and 
accurately through the TSVD method with the 5\% and 10\% noise level respectively.

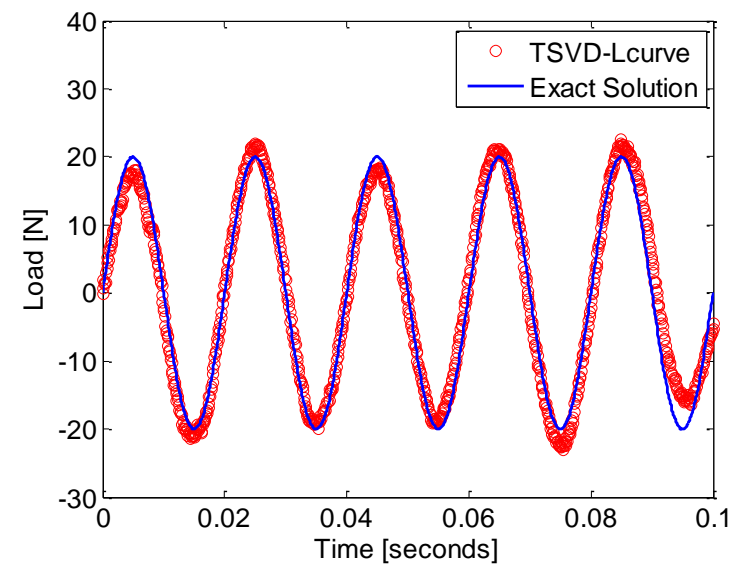

(a)

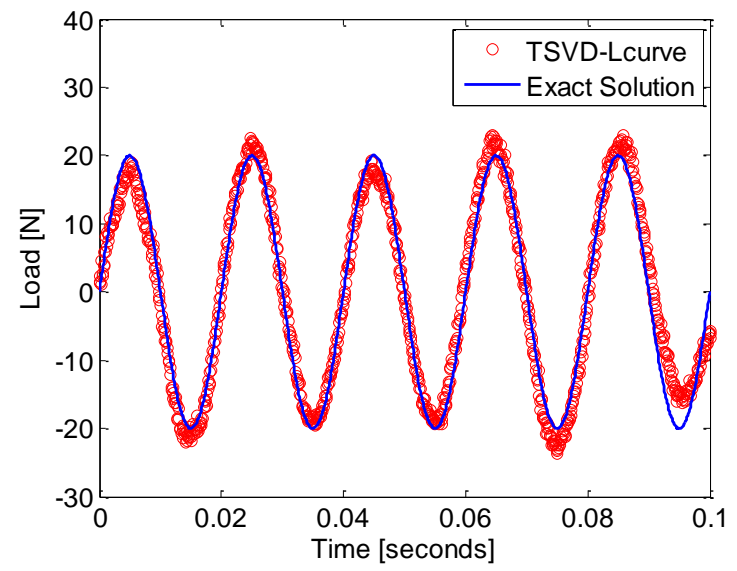

(b)

Fig. 11 Identified results of central point load with two levels,

(a) $5 \%$ noise level, (b) $10 \%$ noise level

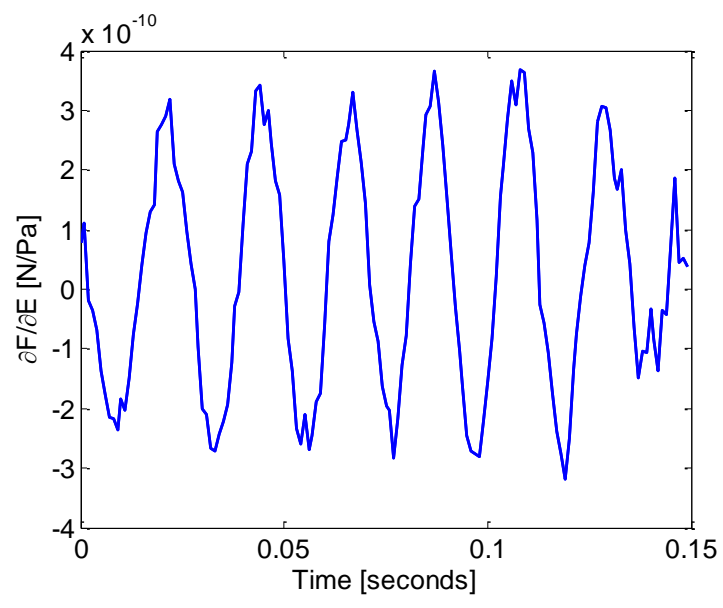

(a)

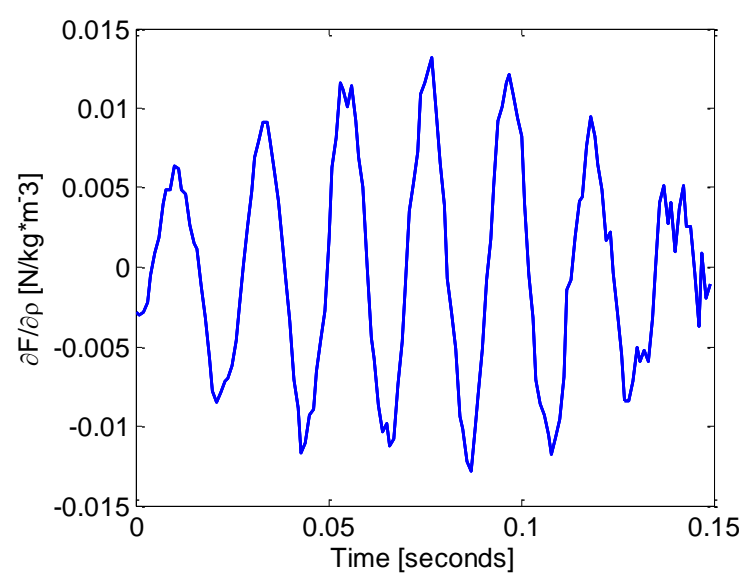

(b)

Fig. 12 Sensitivity analysis of the central point load with respect to the uncertain parameters,

(a) for the Young's modulus, (b) for the density

The sensitivity analyses of the central point load with respect to the Young's modulus and the structural density are shown in Fig. 12. It is obvious that the two ellipsoid parameters affect the load in different trend, i.e. in the first time cycle, the sensitivity curve of the Young's modulus accords with the cosine curve while the sensitivity curve of the 
density conforms to the sine curve. Furthermore, the variation of the two different parameters influences the load in different magnitude level.

Considering the different noise levels and uncertainty level, the dynamic load ranges of the excitation point are respectively computed according to the ellipsoid model and interval model and shown in Figs. 13 and 14.

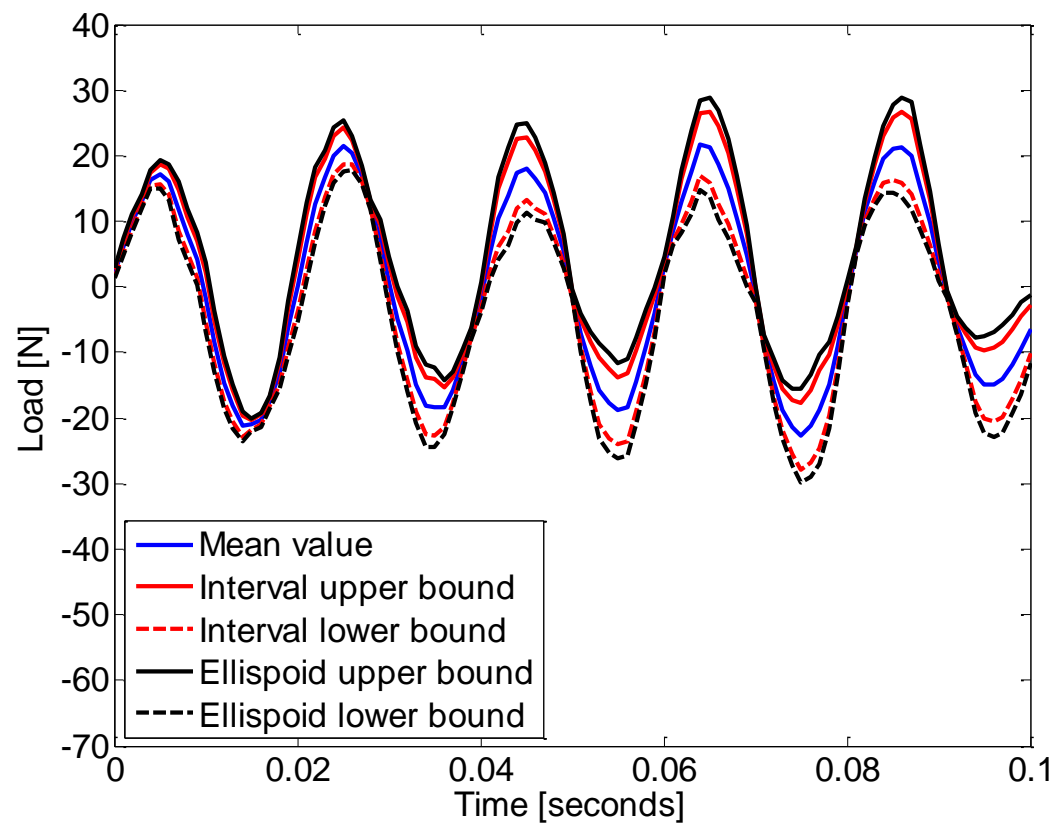

Fig. 13 Comparison between the two methods with 5\% noise and 5\% uncertainty levels 


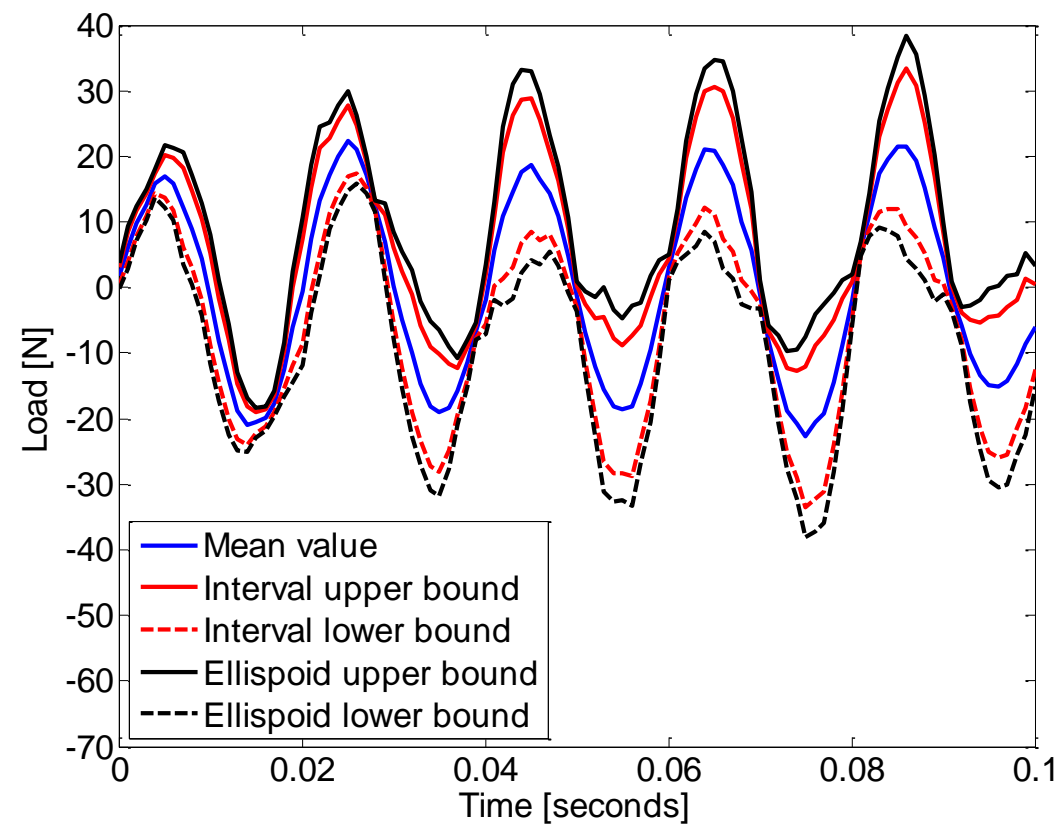

Fig. 14 Comparison between the two methods with $10 \%$ noise and $10 \%$ uncertainty levels

Figs. 13 and 14 show that the upper and lower bounds of the load can be identified effectively through the non-probabilistic methods. With the increase of the uncertainty level, the relative variability computed through the convex method is larger than that computed by the interval method as shown in Figs. 13 and 14. The relative variability of the bounds calculated by convex methods in each time point contain those calculated by interval methods, which indicates that the convex ellipsoid method is more inclusive. This implies that the convex ellipsoid model is more reliable than the interval method. The results based on the former provide more redundancy for the structure design, while the latter makes the range of the identified results narrower.

\subsection{A vehicle roof coupled with the interior cavity}

The vehicle roof used to cover the passenger compartment is the headliner component of the body and it can provide the stiffness contribution for the whole body. Considering 
the light-weight and low-rigidity property of the thin-walled roof structure, the passenger compartment cavity may produce acoustic noise due to the coupling effect between the roof and the cavity.

Here the load identification in the interaction between the thin-walled structural roof and the passenger cavity is investigated, as illustrated in Fig. 15. The passenger cavity filled with fluid air and occupied by the seats is considered as the acoustic part. The exciting force $F=80 \sin (500 \pi t)$ is acting on the middle point (marked as R) of the roof which is selected as the load to be identified. The acoustic response of the driver's left ear (marked as A) is treated as the virtual measurement under the enforced excitation $F$. The four sides surrounding the roof are fixed, the top of the passenger compartment is considered to interact with the roof, while the remaining surfaces of the cavity are treated as the rigid wall. The roof with thickness $0.8 \mathrm{~mm}$ is discretized into 340 four-node shell elements containing 373 nodes, while the acoustic cavity is discretized into 46,828 four-node tetrahedron elements containing 9,718 nodes. The nodal spacing of the acoustic mesh is $0.1 \mathrm{~m}$. 


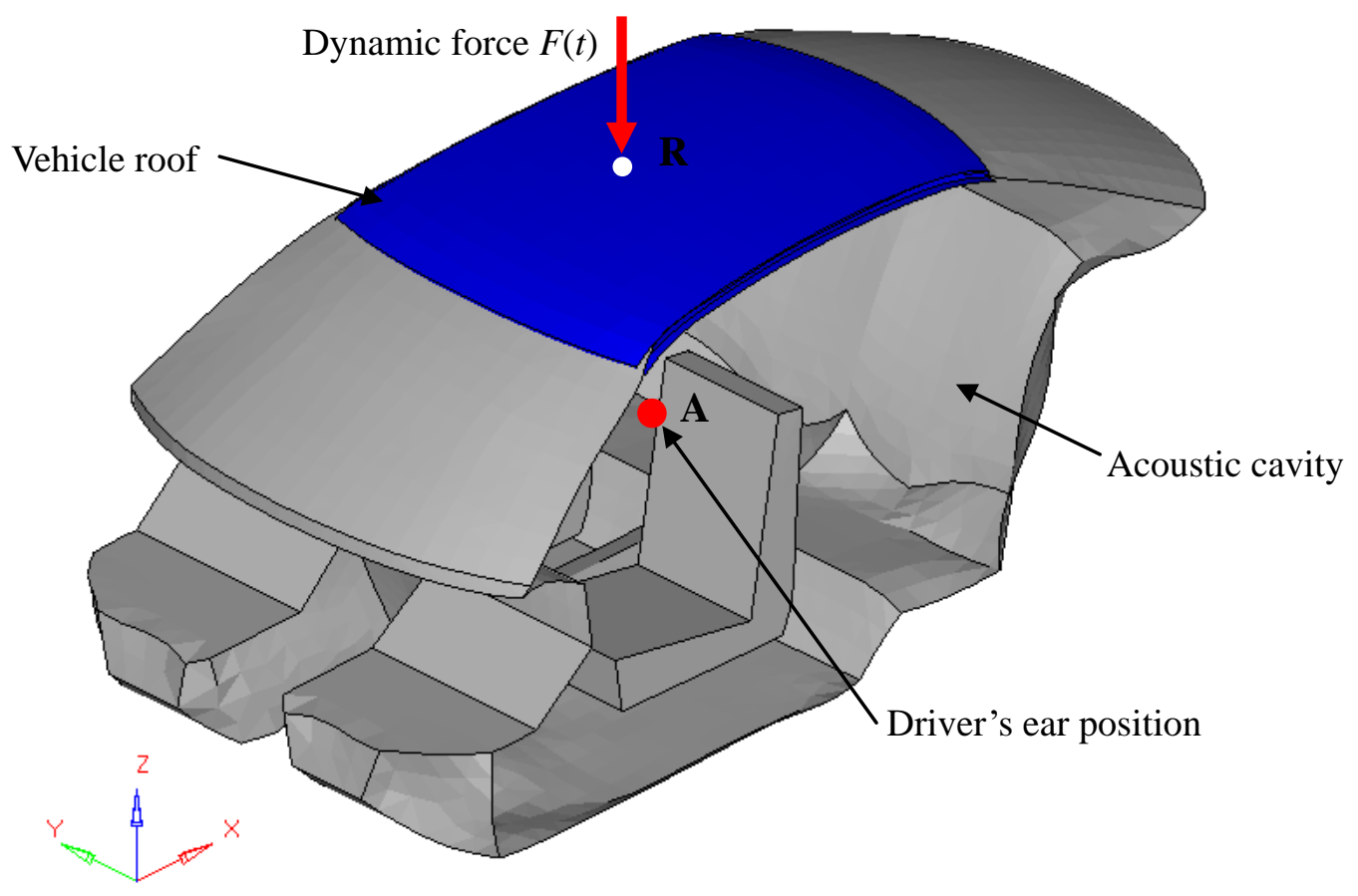

Fig. 15 The 3D model of the roof coupled with the interior cavity

The properties of the structural material in the simulation are described by the Poisson's ratio $v=0.3$ and the structural damping factor $\zeta=0.06$. In this example, the Young's modulus and the density property of the roof are treated as the non-probabilistic parameters. Two uncertainty levels namely 5\% and 10\% are studied for both the ellipsoid model and interval model, the variation ranges (Table 2) are the same as the second example. The sound velocity and mass density are $c=340 \mathrm{~m} / \mathrm{s}$ and $\rho_{a}=1.225 \mathrm{~kg} / \mathrm{m}^{3}$ respectively.

The central point load identified through pressure-based method using TSVD with L-curve criterion considering the two noise levels is shown in Fig. 16. 


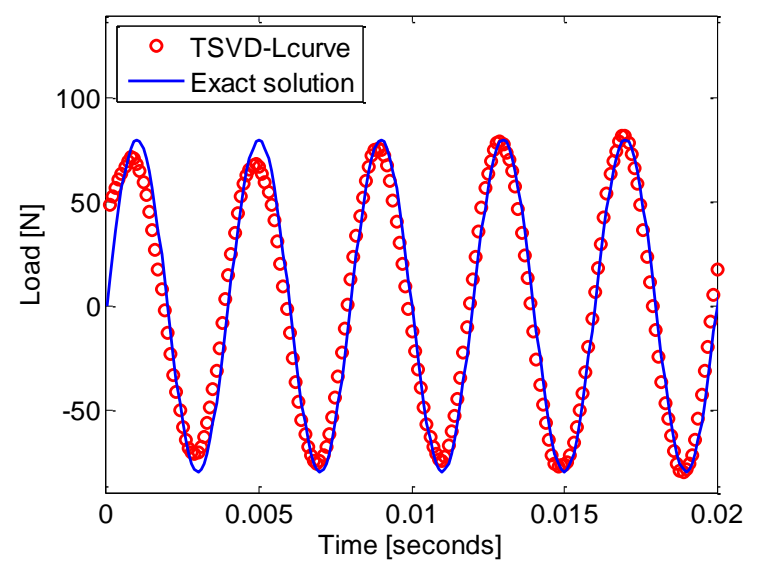

(a)

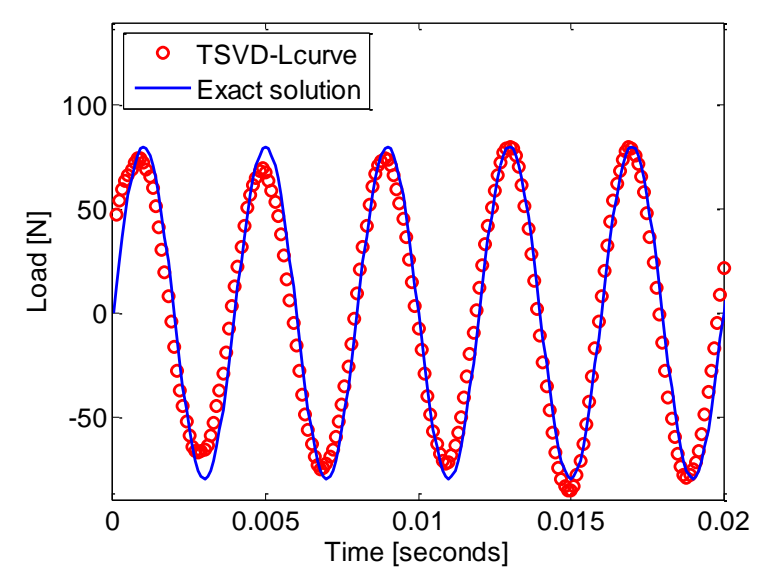

(b)

Fig. 16 Central point load identified results. (a) 5\% noise level, (b) $10 \%$ noise level

It can be found from Fig. 16 that very good agreements between the identified midpoint load and exact load are observed when the noise level increases from 5\% to $10 \%$. It is clearly observed that the load acting on the roof can be effectively identified in the practical engineering model based on the pressure-based method. In the following, in order to quantify the influence produced by the uncertainty, the sensitivity analysis (as plotted in Fig. 17) of the central point load with respect to the uncertain parameters is calculated.

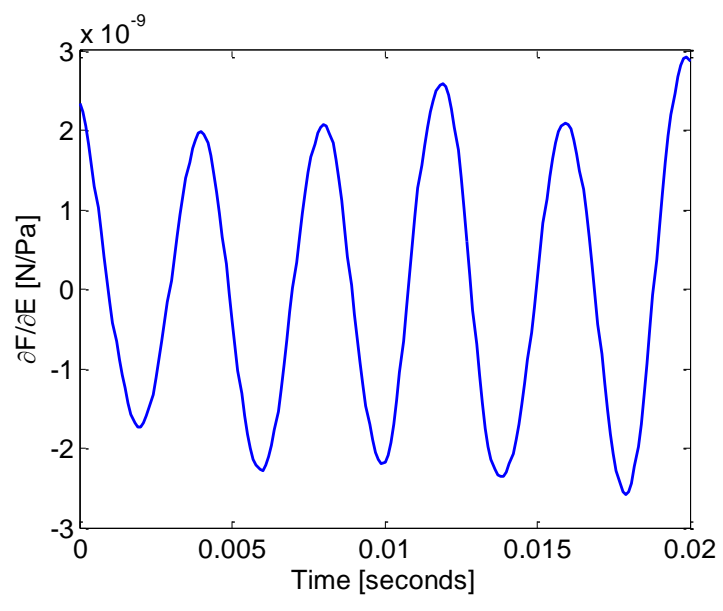

(a)

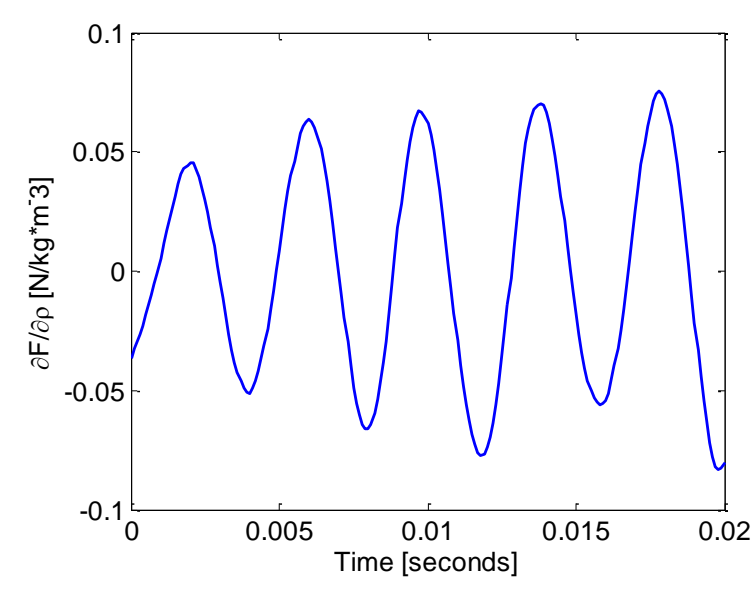

(b)

Fig. 17 Sensitivity analysis of the central point load with respect to the uncertain parameters. 
(a) for the Young's modulus, (b) for the density

Considering the two kinds of noise levels and two kinds of uncertainties, the dynamic load ranges are respectively identified by the ellipsoid model and interval model as plotted in Figs. 18 and 20. The bounds comparison of the load range curves between the two non-probabilistic models is also given in Figs. 19 and 21.

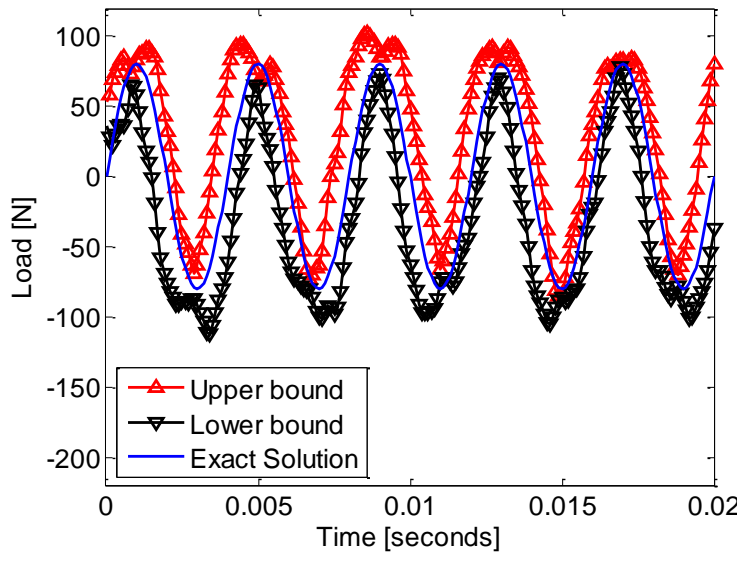

(a)

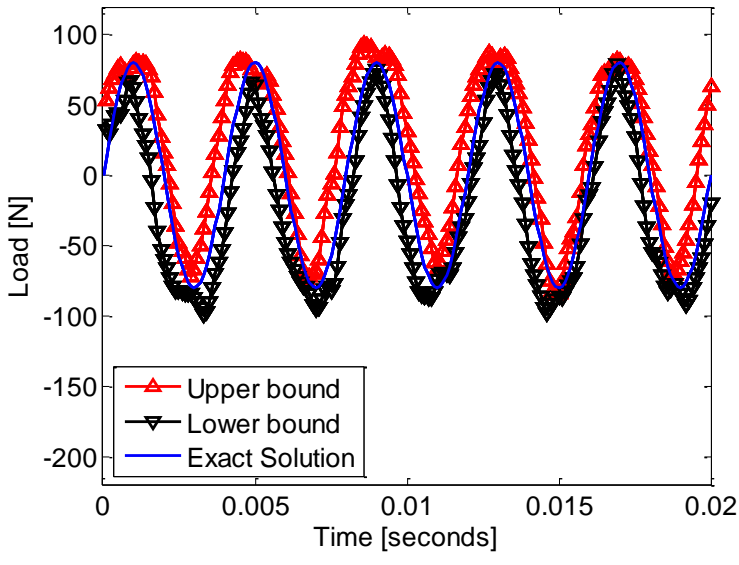

(b)

Fig. 18 The bounds of the identified load with 5\% noise and 5\% uncertainty levels based on the two non-probabilistic methods. (a) Ellipsoid method, (b) Interval method

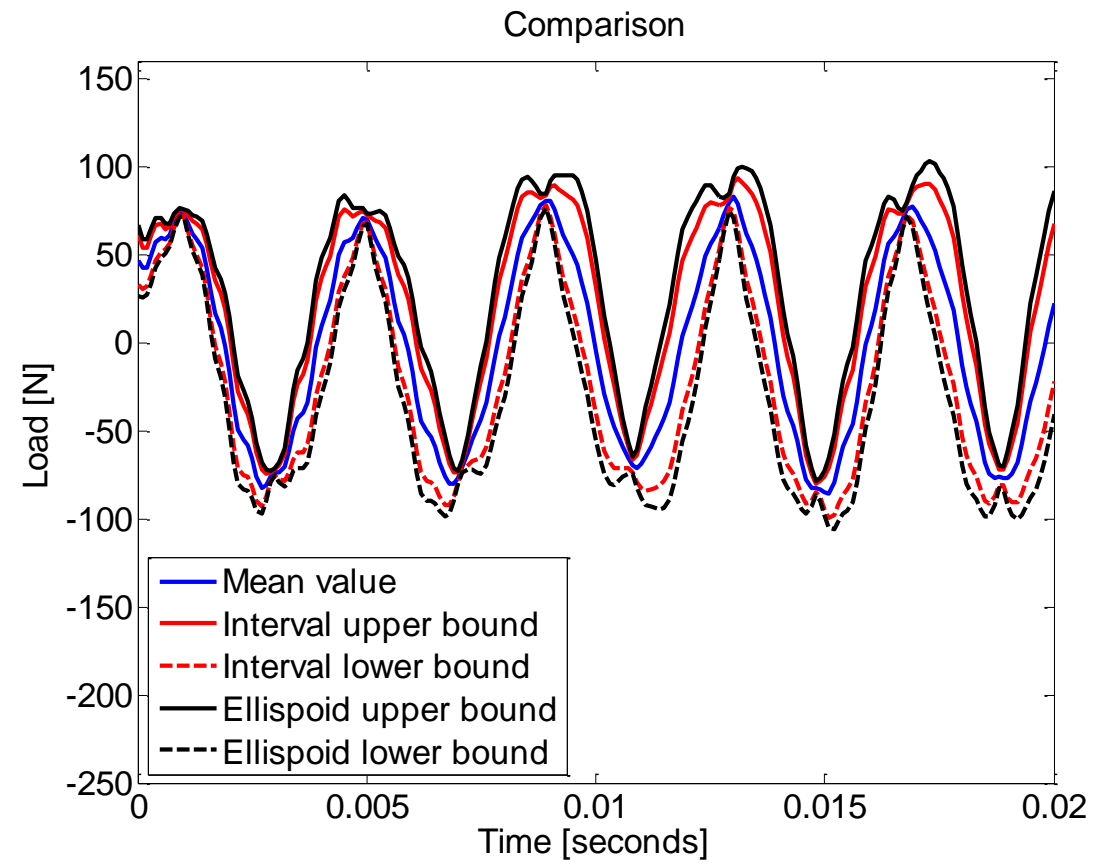


Fig. 19 Comparison between the two methods with 5\% noise and 5\% uncertainty levels

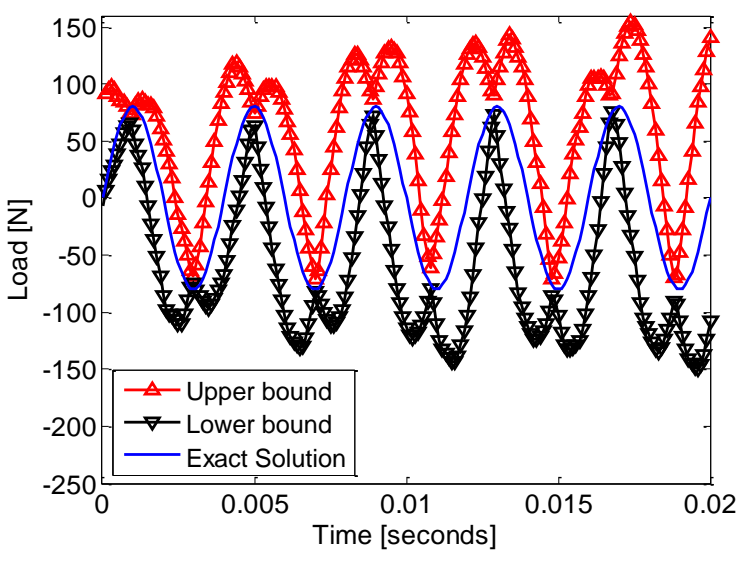

(a)

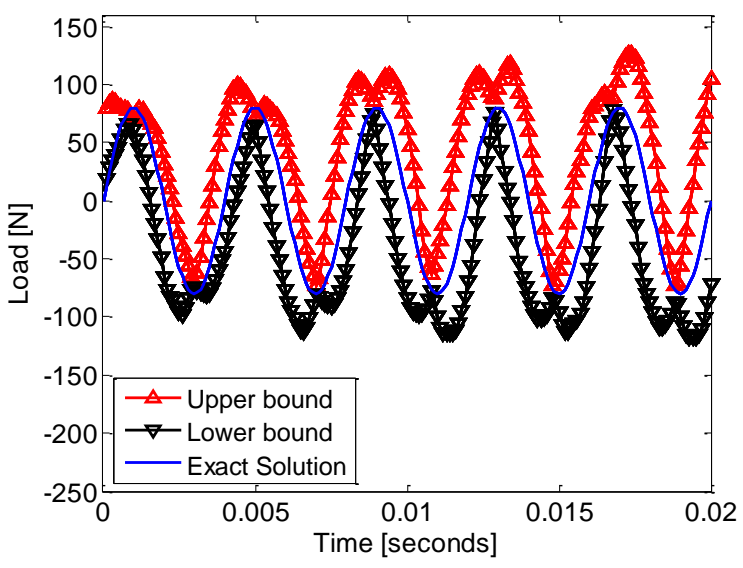

(b)

Fig. 20 The bounds of the identified load with $10 \%$ noise and $10 \%$ uncertainty levels based on the two non-probabilistic methods. (a) Ellipsoid method, (b) Interval method,

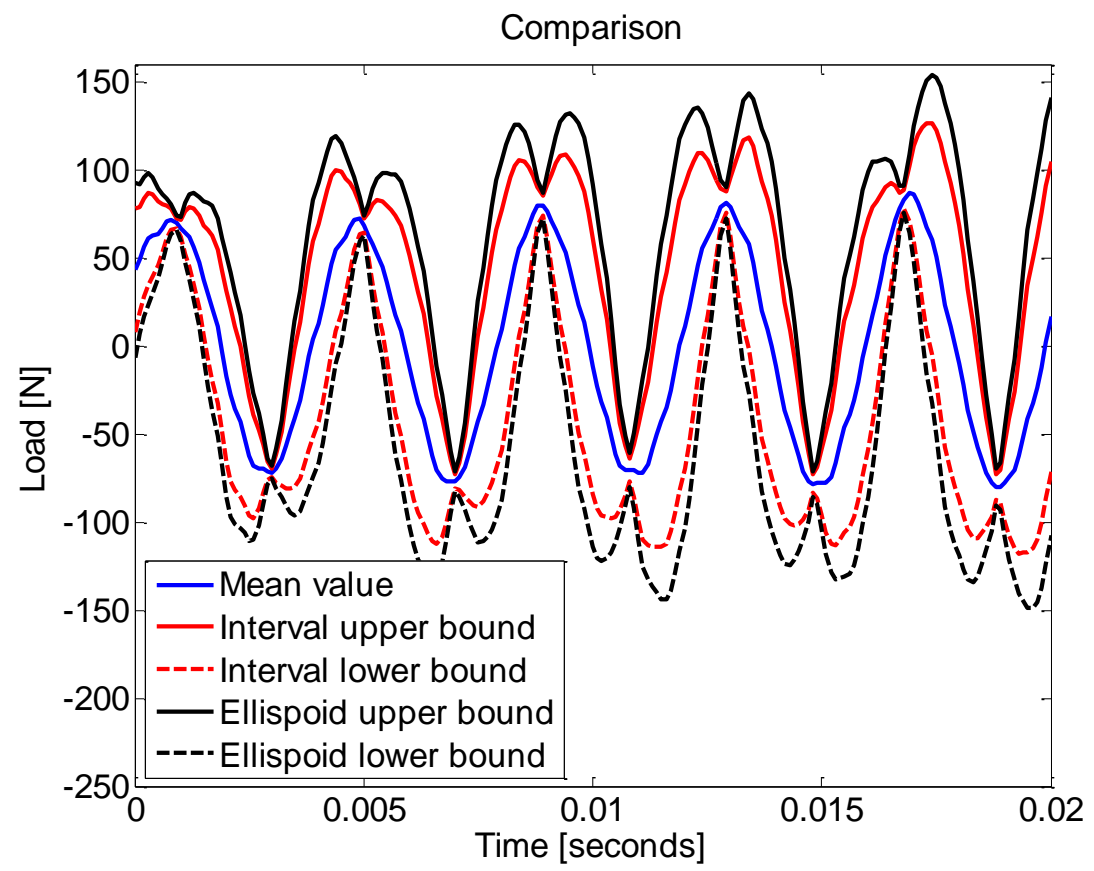

Fig. 21 Comparison between the two methods. with $10 \%$ noise and $10 \%$ uncertainty levels

As outlined in Figs. 18 and 20, the upper and lower bounds of the load can be identified stably in all the time range according to the pressure-based method combined 
with the non-probabilistic method. It is noted that the bounds derived from the convex method still contain those from the interval model as shown in Figs. 19 and 21. Therefore, the convex ellipsoid method can provide more redundancy and reliability for engineers as demonstrated in the numerical applications. In addition, it is noticed that the relative variability between the upper and lower bounds become bigger when the uncertainty varies from $5 \%$ to $10 \%$, which indicates that the uncertainty level is one of the controlling factors of the force bounds.

\section{Conclusion}

This paper studies the load identification problems which contain the ASI system uncertainty and the requirement of non-contact measurement of the responses. By using the non-probabilistic analysis models, the predictions of the load bounds are transformed into the identification of the central point load and the sensitivity analyses of the load with respect to the uncertain parameters. The central point load shares a very good agreement with the exact solution using the truncated single value decomposition approach, which is capable of overcoming the ill-posedness of the inverse process when the non-contact acoustic pressure is polluted. The use of acoustic pressure provides great convenience for load identification in the application where non-contact measurement is preferred. The sensitivity analyses of the loads reveal that the Young's modulus and the structure density affect the central point load in different trends and magnitudes. The correctness of the ellipsoid model and interval model has clearly demonstrated that the uncertainty of load 
identification in the ASI system can be well quantified by the non-probabilistic models.

The results also show that the ellipsoid model is more reliable than the interval method as the results based on the former provides more redundancy, while the latter makes the identified results narrow.

\section{Acknowledgements}

The Project is supported by the Foundation for Innovative Research Groups of the National Natural Science Foundation of China (Grant No. 51621004), and the Opening Project of Guangxi Key Laboratory of Automobile Components and Vehicle Technology, Guangxi University of Science and Technology (No. 2017GKLACVTKF01) and the Natural Science Foundation of Hunan Province, China(Grant No. 2017JJ2059).

\section{References}

[1] Wang M, Huang Q. A new hybrid uncertain analysis method for structural-acoustic systems with random and interval parameters. Computers \& Structures. 2016;175:15-28. [2] Liu J, Sun X, Han X, Jiang C, Yu D. Dynamic load identification for stochastic structures based on Gegenbauer polynomial approximation and regularization method. Mechanical Systems \& Signal Processing. 2015;s 56-57:35-54.

[3] Thite AN, Thompson DJ. The quantification of structure-borne transmission paths by inverse methods. Part 1: Improved singular value rejection methods. Journal of Sound \& Vibration. 2003;264:411-31.

[4] Thite AN, Thompson DJ. The quantification of structure-borne transmission paths by inverse methods. Part 2: Use of regularization techniques. Journal of Sound \& Vibration. 2003;264:433-51.

[5] Turco E. A strategy to identify exciting forces acting on structures. International Journal for Numerical Methods in Engineering. 2010;64:1483-508.

[6] Lage YE, Maia NMM, Neves MM, Ribeiro AMR. Force identification using the concept of displacement transmissibility. Journal of Sound \& Vibration. 2013;332:1674-86.

[7] Liu GR, Ma WB, Han X. An inverse procedure for identification of loads on composite laminates. Composites Part B. 2002;33:425-32.

[8] Lu ZR, Law SS. Identification of system parameters and input force from output only. Mechanical Systems \& Signal Processing. 2007;21:2099-111. 
[9] Liu J, Meng X, Zhang D, Jiang C, Han X. An efficient method to reduce ill-posedness for structural dynamic load identification. Mechanical Systems \& Signal Processing. 2017;95:273-85.

[10] Liu J, Sun X, Han X, Jiang C, Yu D. A novel computational inverse technique for load identification using the shape function method of moving least square fitting. Computers \& Structures. 2014;144:127-37.

[11] Giansante N, Jones R, Calapodas NJ. Determination of In-Flight Helicopter Loads. Journal of the American Helicopter Society. 1982;27:58-64.

[12] Hillary B, Ewins DJ. The use of strain gauges in force determination and frequency response function measurements. 1984.

[13] Yen CS, Wu E. On the Inverse Problem of Rectangular Plates Subjected to Elastic Impact, Part II: Experimental Verification and Further Applications. Journal of Applied Mechanics. 1995;62:699.

[14] Li E, He Z, Xu X, Liu G. Hybrid smoothed finite element method for acoustic problems. Comput Method Appl M. 2015;283:664-88.

[15] Li E, He Z, Jiang Y, Li B. 3D mass-redistributed finite element method in structural-acoustic interaction problems. Acta Mechanica. 2016;227:857-79.

[16] Arora V, Wijnant YH, Boer AD. Acoustic-based damage detection method. Applied Acoustics. 2014;80:23-7.

[17] Veronesi WA, Maynard JD. Nearfield acoustic holography (NAH) II. Holographic reconstruction algorithms and computer implementation. Journal of the Acoustical Society of America. 1987;81:1307-22.

[18] Manesh MK, Hematiyan MR, Eghtesad M, Necsulescu DS, Rezazadeh A. Vibrating Loads Identification Using Inverse Acoustics in Fluid-Structure Interaction. Advanced Materials Research. 2012;433-440:51-7.

[19] Arteaga IL, Scholte R, Nijmeijer H. Improved source reconstruction in Fourier-based Near-field Acoustic Holography applied to small apertures. Mechanical Systems \& Signal Processing. 2012;32:359-73.

[20] Deng JH, Gu CS, Liu XD, Li HG. Sound source identification and contribution analysis based on acoustic array technology. Journal of Vibration Engineering. 2010.

[21] Lee CM, Xu Y. A modified transfer matrix method for prediction of transmission loss of multilayer acoustic materials. Journal of Sound \& Vibration. 2009;326:290-301.

[22] Angell TS, Jiang X, Kleinman RE. A distributed source method for inverse acoustic scattering. Inverse Problems. 1997;13:531.

[23] Xia B, Yu D, Liu J. Hybrid uncertain analysis of acoustic field with interval random parameters. Computer Methods in Applied Mechanics \& Engineering. 2013;256:56-69.

[24] Gobbato M, Kosmatka JB, Conte JP. A recursive Bayesian approach for fatigue damage prognosis: An experimental validation at the reliability component level. Mechanical Systems \& Signal Processing. 2014;45:448-67.

[25] Polydorides N. A stochastic simulation method for uncertainty quantification in the linearized inverse conductivity problem. International Journal for Numerical Methods in Engineering. 2012;90:22-39. 
[26] Jin B. Fast Bayesian approach for parameter estimation. International Journal for Numerical Methods in Engineering. 2008;76:230-52.

[27] Song Q, Chissom BS. Fuzzy time series and its models: Elsevier North-Holland, Inc.; 1993.

[28] Hui L, Wen-Bin S, Dejie Y. Uncertainty quantification of squeal instability under two fuzzy-interval cases. Fuzzy Sets and Systems. 2017.

[29] Jiang C, Ni BY, Han X, Tao YR. Non-probabilistic convex model process: A new method of time-variant uncertainty analysis and its application to structural dynamic reliability problems. Computer Methods in Applied Mechanics \& Engineering. 2014;268:656-76.

[30] Elishakoff I, Elettro F. Interval, ellipsoidal, and super-ellipsoidal calculi for experimental and theoretical treatment of uncertainty: Which one ought to be preferred? International Journal of Solids \& Structures. 2014;51:1576-86.

[31] Cao HJ, Duan BY. Approach on the non-probabilistic reliability of structures based on uncertainty convex models. Chinese Journal of Computational Mechanics. 2005;22:546-9+78.

[32] Impollonia N, Muscolino G. Interval analysis of structures with uncertain-but-bounded axial stiffness. Computer Methods in Applied Mechanics \& Engineering. 2011;200:1945-62.

[33] Wang X, Yang H, Wang L, Qiu Z. Interval Analysis Method for Structural Damage Identification Based on Multiple Load Cases. Journal of Applied Mechanics. 2012;79:1010.

[34] Qiu Z, Ma L, Wang X. Non-probabilistic interval analysis method for dynamic response analysis of nonlinear systems with uncertainty. Journal of Sound \& Vibration. 2009;319:531-40.

[35] Liu J, Han X, Jiang C, Ning HM, Bai YC. Dynamic load identification for uncertain structures based on interval analysis and regularization method. International Journal of Computational Methods. 2011;8:667-83.

[36] Zhang GY, Lu H, Yu DP, Bao ZM, Wang HY. A node-based partly smoothed point interpolation method (NPS-PIM) for dynamic analysis of solids. Engineering Analysis with Boundary Elements. 2018;87:165-72.

[37] Zhang GY, Li Y, Gao XX, Hui D, Wang SQ, Zong Z. Smoothed Point Interpolation Method for Elastoplastic Analysis. International Journal Of Computational Methods. 2015;12:12.

[38] Zhang GY, Wittek A, Joldes GR, Jin X, Miller K. A three-dimensional nonlinear meshfree algorithm for simulating mechanical responses of soft tissue. Engineering Analysis with Boundary Elements. 2014;42:60-6.

[39] Li E, He Z, Xu X, Liu G, Gu Y. A three-dimensional hybrid smoothed finite element method (H-SFEM) for nonlinear solid mechanics problems. Acta Mechanica. 2015;226:4223-45.

[40] Li E, He Z, Liu G. Evaluation of the stiffness matrix in static and dynamic elasticity problems. Acta Mechanica. 2018;229:363-88. 
[41] Yin S, Yu D, Yin H, Xia B. Interval and random analysis for structure-acoustic systems with large uncertain-but-bounded parameters. Computer Methods in Applied Mechanics \& Engineering. 2016;305:910-35.

[42] Liu G-R, Han X. Computational inverse techniques in nondestructive evaluation: CRC press; 2003.

[43] Hansen PC, O'Leary DP. The use of the L-curve in the regularization of discrete ill-posed problems. SIAM Journal on Scientific Computing. 1993;14:1487-503.

[44] Qiu Z. Convex models and interval analysis method to predict the effect of uncertain-but-bounded parameters on the buckling of composite structures. Computer Methods in Applied Mechanics \& Engineering. 2005;194:2175-89.

[45] Liu Y, Shepard WS. Dynamic force identification based on enhanced least squares and total least-squares schemes in the frequency domain. Journal Of Sound And Vibration. 2005;282:37-60. 